\title{
Autour d'une mosaïque médiévale de la Grande Mosquée de Damas
}

A Medieval Mosaic in the Great Mosque of Damascus

$$
\text { عن الفسيفساء القروسطية للجامع الكبير بدمشق }
$$

\section{François Bogard}

\section{OpenEdition \\ Journals}

Édition électronique

URL : http://journals.openedition.org/beo/851

DOI : $10.4000 /$ beo.851

ISBN : 978-2-35459-347-9

ISSN : 2077-4079

Éditeur

Presses de l'Institut français du Proche-Orient

Édition imprimée

Date de publication : 1 décembre 2012

Pagination : 91-122

ISBN : 978-2-35159-379-0

ISSN : 0253-1623

Référence électronique

François Bogard, «Autour d'une mosaïque médiévale de la Grande Mosquée de Damas », Bulletin d'études orientales [En ligne], Tome LXI | décembre 2012, mis en ligne le 11 mars 2013, consulté le 03 mai 2019. URL : http://journals.openedition.org/beo/851 ; DOI : 10.4000/beo.851 


\section{Autour d'une mosaïque médiévale de la Grande Mosquée de Damas}

François BOGARD

La mosaïque médiévale ${ }^{1}$ qui orne intérieurement le pignon de la nef axiale de la Grande Mosquée de Damas présente plusieurs groupes d'architectures sur un fond végétal. L'œuvre semble inspirée par les panneaux d'époque omeyyade, mais en diffère également beaucoup. Marguerite Van Berchem ${ }^{2}$ a proposé de dater ce panneau du règne de Malikšāh à la fin du XI ${ }^{\mathrm{e}}$ siècle, sur la foi de travaux attestés ${ }^{3}$ par une épigraphie qui ne mentionne pas les mosaïques, et sur des critères stylistiques qui semblent aujourd'hui fragiles.

Pour reprendre la problématique de la datation de cette mosaïque, nous tenterons tout d'abord de déterminer les sources auxquelles l'image produite se réfère, d'une part, par l'examen de l'iconographie de l'Antiquité tardive et de la période omeyyade, et d'autre part, par celle de la fin de la période médiévale que l'on retrouve par exemple dans les livres illustrés du début du XIII ${ }^{\mathrm{e}}$ siècle et les mosaïques du début de la période mamelouke. L'objectif sera ainsi de proposer une datation pour la confection de ce panneau. Une fois cette identification réalisée, nous élargirons notre propos et nous chercherons à interpréter la signification de la réalisation d'une telle image dans le cadre plus général de la production médiévale.

\section{La situation du panneau dans la Grande Mosquée de Damas}

On sait que la Grande Mosquée a connu de nombreux incidents (incendies et tremblements de terre) qui ont occasionné des travaux de réparation, voire de reconstruction. Certains de ces travaux sont datés par des inscriptions, d'autres connus

\footnotetext{
1. VAN BERCHEM 1969, p. 354-363.

2. VAN BERCHEM 1969, p. 323-372.

3. L'architecte de Tutuš entreprend la reconstruction de la coupole sur une nouvelle échelle, entraînant un remaniement global de la nef axiale, et notamment de ses supports. Les modestes vestiges des mosaïques des piliers de la coupole n'ont pas le même caractère que celle qui nous préoccupe et datent vraisemblablement de l'époque de Malikšāh. Voir VAN BERCHEM 1969, p. 323-372. De grandes plaques de marbre conservées au Musée national de Damas portent des inscriptions qui les commémorent. Voir RCEA, VII, n²734, 2735, 2736, 3737 (475/1082 AD) ; AL-'UsH 1976, p. 254, fig. 145 .
} 
par des textes historiques, d'autres encore seulement par leur découverte ou leur étude. Le mur nord de la cour fit l'objet d'une reconstruction au début du XII siècle attestée par les inscriptions lapidaires qui occupent aujourd'hui encore le linteau des portes et fenêtres qui y sont percées ${ }^{4}$. Certains décors de mosaïque de la cour (fig. 4-5) datent de l'époque de Nūr al-Dīn ${ }^{5}$, d'autres de l'époque de Baybars ${ }^{6}$.

La salle de prière est marquée par l'implantation d'une nef axiale, dont les deux extrémités se terminent par un mur pignon coiffé d'un fronton triangulaire (fig. 1). Un décor de mosaïque exceptionnel par son sujet et ses dimensions orne la partie supérieure de l'intérieur du mur pignon de la façade sur cour de cette nef tandis que la partie basse de la paroi reste ornée de motifs omeyyades. Le thème du décor est une série de bâtiments représentée dans un environnement végétal. Ces architectures se distinguent nettement des réalisations omeyyades ${ }^{7}$, mais aussi de celles que l'on attribue à Baybars dans une partie du portique ouest ${ }^{8}$, et de celles que fit exécuter pour le mausolée de ce dernier son successeur Qalāwūn (fig. 22) ${ }^{9}$.

Ce panneau, très altéré dans son état actuel ${ }^{10}$, constitue l'encadrement du grand arc en défoncement sous lequel s'ouvrent, extérieurement (fig. 1) comme intérieurement, deux étages de baies sur la cour, c'est-à-dire une triple colonnade sur des bases surhaussées, surmontée de trois fenêtres en plein cintre dont celle du centre, dans un souci d'harmonisation avec la courbe de l'arcature aveugle est plus haute. Il fait face au mihrāb, et se trouve dans la travée immédiatement voisine de la célèbre Coupole de l'Aigle (Qubba al-Nasr), construite en 1082 sous le règne de Malikšāh.

\section{Iconographie de la mosaïque}

L'image qui occupe l'encadrement de l'arc présente un paysage arboré où se déploient des architectures ${ }^{11}$. La composition (fig. 3 ) comporte de grandes lacunes notamment dans les écoinçons et dans la partie droite, mais la partie supérieure conserve un groupe identifiable composé, selon Marguerite Van Berchem, à l'ouest (à gauche) d'une « mosquée » (fig. 2, A), que l'on reconnaît à sa grande coupole précédée d'une série d'arcades, et, à

4. Voir AlLEN 2003, et plus spécifiquement le chapitre 1 intitulé « Damascus Before Nūr al-Dīn, Doorways of The Great Mosque».

5. Inscription au nom de Nūr al-Dīn au-dessus d'une arcade du portique oriental de la cour, près de l'angle nord-est.

6. VAN BERCHEM 1969, fig. 406 ; Finster 1970-71, fig. 33, 65 ; De LOREY 1931, p. 345-347.

7. Pour comparaison purement formelle, voir DE LOREY 1931, pl. LXV, LXVII, LXXII ; FINSTER 1970-71.

8. De Lorey 1931, p. 345-347 ; VAn Berchem 1969, fig. 406.

9. Flood 1997, p. 57-79; RABBAT 1997-99, p. 2-5.

10. À l'intérieur de la salle de prière, la restauration n'a pas tourné à la reconstitution comme à l'extérieur. Sans doute parce qu'une telle reconstitution nécessiterait une compréhension de l'ensemble qui reste difficile. Nous verrons en le décrivant qu'il y a d'importantes lacunes, mais que les motifs les plus significatifs ont été préservés. Seule intervention récente, un bandeau peint a été réalisé récemment pour régulariser la partie supérieure de ce panneau et faire la jonction avec le plafond en bois qui le surplombe.

11. Les lettres A, B, C, D renvoient à la partie concernée du croquis repris de celui de Michel Écochard. La partie basse de la mosaïque, du côté gauche n'est pas répertoriée dans ce croquis. 
l'est (à droite), d'un « palais » (fig. 2, D) ${ }^{12}$. Nous retiendrons cette terminologie. Une série d'édifices prend place entre ces deux pôles au sommet de l'arc (fig. 2, B et C). Enfin, le côté occidental conserve des représentations d'autres constructions difficilement identifiables le long de l'arc, disséminées dans la végétation de l'écoinçon.

La façade de la mosquée (fig. 2, A) est disposée horizontalement et est composée d'une colonnade sous une suite ininterrompue de merlons en « $\mathrm{T}$ » placés tête-bêche qui évoque des décors en jeux de briques (fig. 2, détail 1). Trois arcades étroites ${ }^{13}$ sont disposées de part et d'autre d'un grand arc central en un motif symétrique ${ }^{14}$ amputé du côté droit par la découpe, pourtant préexistante, du grand arc en défoncement souligné d'un tore très proéminent qui constitue la limite intérieure du cadre de l'image. Le mosaïste n'en a pas tenu compte en installant son dessin (fig. 8). Cette gestion du motif laisse percevoir une certaine maladresse, et peut-être l'existence d'un modèle que l'on recopie plus ou moins fidèlement, sans savoir l'adapter parfaitement au cadre ${ }^{15}$.

La grande arcade centrale de la mosquée s'inscrit dans un encadrement rectangulaire aux écoinçons doublés d'un bandeau qui les individualise. Elle représente probablement l'arcature élargie d'une nef axiale ${ }^{16}$, ouverte d'une porte en plein cintre au travers de laquelle nous voyons une colonnade intérieure de l'édifice que deux segments d'arcs retombant sur une colonne centrale indiquent. Ce procédé permet aux artistes ${ }^{17}$ de suggérer au moins une autre nef parallèle au mur de qibla. Les arcades latérales sont sans doute celles de la salle de prière, car le couronnement de merlons de la façade ne s'interrompt pas et la toiture se greffe sur l'ensemble de cette frise. Il ne semble donc pas que l'on doive envisager les ailes d'un monument, mais bien la continuité d'une colonnade de façade, et plus probablement celle de la salle de prière donnant sur la cour. Le mur de clôture de la mosquée, lui, a été supprimé selon la formule que Paul Lampl, dans une étude sur les schémas de représentation des architectures, a baptisée «Open Wall » ${ }^{18}$. Cela permet de mettre en évidence les éléments caractéristiques de l'édifice, ici, une mosquée hypostyle. Enfin, chacune des arcades latérales est timbrée d'une succession verticale de fleurons, chacun naissant du précédent ${ }^{19}$.

12. VAN BERCHEM 1969, p. 323-372.

13. La troisième à droite se perd dans un élément végétal dans la partie haute.

14. Il ne reste à gauche qu'une partie de deux colonnes qui séparaient au moins trois arcades.

15. On peut rapprocher la gestion de cet ensemble de l'un des écoinçons publiés par De Lorey, mais dans ce dernier, une perspective a été mise en place pour éviter ce type d'amputation, alors que nous avons ici une vue délibérément frontale pour le premier plan. DE LOREY 1931, pl. LXXII.

16. On ne peut cependant pas écarter l'hypothèse qu'il s'agit d'un portail ouvert sur l'extérieur.

17. On trouve déjà ce procédé employé dans la Grande Mosquée, dès l'époque omeyyade : voir ETTINGHAUSEN 1977, illustration p. 27 ; mais aussi dans l'art du livre, par exemple, p. 106 (Manuscrit de Leningrad/Saint Petersbourg des Maqāmāt d'al-Harīīi), ou, plus proche par le sujet de notre mosaïque : BARRUCAND 1994, p. 87, fig. 78. (page 41 du manuscrit de Saint-Petersbourg- Leningrad, S 23).

18. LAMPL 1961, p. 9-10; Duval 2003, p. 219.

19. Ces tiges de fleurons remplacent peut-être les personnages d'un modèle chrétien. Plus simplement, les mosaïques de la Mosquée des Omeyyades comportent des arcades où prennent place des feuillages de vigne enroulés autour des colonnes, voir DE LOREY 1931, pl. LVII. Ce motif a pu inspirer un mosaïste médiéval qui a préféré le fleuron à la feuille de vigne. 
Un toit dont les lignes convergent vers l'arrière-plan rejoint cette colonnade à une grande coupole côtelée qui est posée sur deux niveaux percés de fenêtres, plein cintre au niveau bas avec de petits oculi dans les écoinçons de leurs encadrements, et carrées au niveau supérieur. On peut interpréter ces étages comme deux niveaux de tambours ${ }^{20}$. À mon sens, ce dessin évoque une mosquée hypostyle à nef axiale, pourvue d'une coupole sur la travée centrale avant le mihrāab, dispositif qui est celui de la Grande Mosquée elle-même, qui est largement repris en Syrie et à Damas même ${ }^{21}$.

De chaque côté de la coupole centrale, trois bâtiments étroits, en forme de "tours » à deux étages se succèdent vers l'extérieur, sans symétrie (fig. 2, A et 8). Le bâtiment le plus à droite a reçu un dessin de grands carreaux rectangulaires comme pour indiquer une architecture de pierre en grand appareil ${ }^{22}$ et est couronné de deux coupoles (?) de petites dimensions qui en portent une troisième. Cette superposition est l'un des artifices qui permet de suggérer la profondeur. Bien que nous soyons tentés de chercher dans ces édifices des minarets, car ceux de Damas et de la Syrie médiévale sont carrés de plan et s'accorderaient assez facilement à une représentation sous la forme de ces «tours ", qui sont assez proches de celles que donnent les chrétiens à leurs clochers depuis l'Antiquité tardive et le Haut Moyen Âge ${ }^{23}$, tout nous écarte de cette interprétation : le nombre trop grand des «tours » ${ }^{24}$, l'absence d'une référence claire à cette fonction, et la variété des couronnements le plus souvent incompatibles avec l'image d'un minaret: un fronton sommital, sur l'un de ces édifices, suggère plutôt un toit en bâtière, un toit en terrasse pour un autre et une double coupole (si j'interprète correctement ce motif mutilé) n'ont rien en commun avec ce type de monument.

C'est aux mosaïques omeyyades que l'on va se référer pour interpréter ces « tours ». Richard Ettinghausen a publié la photographie détaillée d'un « village » ${ }^{25}$ où l'on voit des bâtiments élevés, en forme de "tours ", avec des toits en bâtière, d'autres simplement inclinés, des frontons, et des fenêtres hautes et étroites, des portes rectangulaires. Ces maisons sont étagées et s'imbriquent les unes dans les autres, comme celles que l'on voit sur notre mosaïque (fig. 2 et 7), où l'on remarque sur la droite une toiture inclinée à mi-

20. Bien que nous ne sachions rien de précis sur ce point de la coupole construite par Malikšāh, la superposition de deux tambours pour porter une coupole côtelée est la règle à Damas dans l'architecture depuis au moins 1148 . La plus ancienne turba présentant ce dispositif à Damas est la Turba al-Nağmiyya (1148). La plupart des coupoles construites à Damas ensuite comportent ces deux niveaux de transition, avec quelques variantes à partir de l'époque mamelouke (après 1260). Pour ce mausolée, voir ALLEN 2003, chap. 1.

21. À Damas même, le Ğāmi` al-Tawba reprend au XIII ${ }^{\mathrm{e}}$ siècle ce dispositif. D'autres mosquées du XIII ${ }^{\mathrm{e}}$ siècle sont bâties sur un plan proche, mais si la nef axiale est toujours présente, la coupole peut être absente : la mosquée des Hanbalites (al-Șāliḥiyya), par exemple.

22. Un tel quadrillage est visible sur une peinture des muqarnas de la chapelle palatine de Palerme. Cf. ETTINGHAUSEN 1977, p. 49 ; ce quadrillage est souvent la marque de l'architecture militaire, c'est ainsi que sont représentées tours et portes urbaines dans les mosaïques d'Umm al-Rașāṣ en Jordanie (Église Saint-Étienne, viII siècle) et de Tayyibat al-Imām en Syrie. Voir Duval 2003, pour Umm al-Rașāṣ, p. 245-259, fig. 19, pour Tayyibat al-Imām (754 ap. J. C.), p. 243 et fig. 17 b et c.

23. Mosaïques de Saint-Etienne d'Umm al-Rașāṣ, par exemple, au viII siècle, voir Duval 2003, p. 211-285, fig. $19-21$.

24. Et pourtant, la Grande Mosquée de Damas en compte trois, ce qui est exceptionnel en Syrie au Moyen Âge.

25. ETTINGHAUSEN 1977, p. 25. 
hauteur de la «tour », qui forme une excroissance que l'on pourrait interpréter comme une maison basse. La mosquée est donc, en fait, intégrée à un espace urbain.

Ainsi, la mosquée est l'élément central du décor, extrait du milieu urbain comme caractéristique de la ville qui, elle, est symboliquement représentée par cette agglomération de bâtiments.

Un peu plus bas, à gauche, un grand fragment, l'« ensemble F » (fig. 8), a échappé à la dégradation. Plusieurs bâtiments sont placés dans un cadre végétal dominé notamment par un grand arbre qui se rattache complètement à la conception de ceux que les artistes omeyyades ont placés en façade extérieure de cette nef axiale, troncs et branches sont pourvus de feuilles individualisées. Tous ces édifices sont des réinterprétations des motifs secondaires des mosaïques omeyyades, avec les mêmes règles de représentation de la profondeur.

Le groupe B, à la clef, est composé de quatre éléments (fig. 9) : deux arcades centrales, retombant sur trois colonnes, couronnées de merlons dentés qui rappellent ceux des mosquées fatimides du Caire et emplies d'une tige florale formée d'une succession de fleurons issue d'un vase, comparable à celles que nous avons décrites pour les arcades de la mosquée; deux «tours » d'où s'échappent des feuilles d'acanthe ${ }^{26}$ cantonnent ces arcades aux extrémités. Celle de gauche est ouverte par une haute porte pourvue d'un arc en plein cintre surmontée d'une fenêtre aux contours recticurvilignes ${ }^{27}$, celle de droite est ornée d'un grand entrelacs géométrique en un nœud rubané qui se referme sur lui-même. Il est difficile de proposer une fonction à cet ensemble, si ce n'est qu'il s'agit d'une vue qui présente la façade, la porte, et le fond (la paroi au nœud rubané) d'une salle à colonnes. Des buissons disposés de part et d'autre servent de lien végétal entre tous ces groupes architecturaux.

Un édicule sur colonnes (fig. 9, C) prend place entre ce dernier groupe et l'autre élément essentiel de ce décor, le "palais » (fig. 10, D). Marguerite Van Berchem propose d'y reconnaitre la coupole du Trésor (hazna) de la cour de la Grande Mosquée. L'édifice est toutefois pourvu d'un décor étonnant en zigzags, ou de bâtons brisés disposés horizontalement ${ }^{28}$. L'absence de bases pour ces colonnes, l'aspect polygonal, suggéré par les bâtons rompus, de la partie supérieure de la construction, évoque la hazna de Damas et rend cette hypothèse plausible. Néanmoins, le couronnement est en feuilles d'acanthe, parent de la couverture végétale des deux tours du motif central (B) et surtout de nombreux motifs visibles dans la partie omeyyade du décor de la mosquée, ce qui montre une fois encore la limite du réalisme de la représentation ${ }^{29}$.

26. Une interprétation possible de ces végétaux que l'on retrouve régulièrement en couronnement des édifices dans les œuvres omeyyades et postérieures est le souvenir des acrotères de l'architecture de l'Antiquité hellénistique et classique.

27. Cette figure est bien connue dès l'époque fatimide, où elle ponctue, sous forme de cadre, les poutres des palais fatimides. IMA, 1998, p. 88-89, fig. 1-3.

28. On retrouve ce type de zigzags au-dessus de l'inscription $n^{\circ} 2$, voir fig. 9.

29. À moins qu'il n'y ait là une contamination du décor de mosaïques qui couvre le Trésor lui-même? 
Le palais (fig. 10, D), enfin, est très mutilé : le rez-de-chaussée n'est visible que très partiellement sous la forme de deux groupes de deux colonnes (ou deux pilastres ?). Faut-il le concevoir tout entier sur un étage hypostyle ou bien ouvert sur une cour à portiques? Est-il ouvert au centre d'un portail monumental ? Seule certitude, si un tel portail a existé, il est, comme celui de la mosquée, limité en hauteur par la frise, intacte ${ }^{30}$, qui sert d'architrave et de base à deux coupoles côtelées (en pétales) de profil outrepassé posées sur un tambour unique à chaque extrémité. Elles encadrent un pignon doté d'un fronton triangulaire. Deux coupoles non côtelées et sans tambours prennent place dans les espaces intermédiaires entre les trois précédents éléments, des vases à anses les surmontent. Ces coupoles sont probablement destinées à donner une profondeur à une architecture présentée de manière frontale.

Enfin, il reste un groupe de végétaux denses sur la droite (fig. 11), mal relié actuellement à l'ensemble, on n'y distingue aucune architecture.

\section{Une iconographie issue de l'Antiquité tardive?}

Nous avons évoqué à plusieurs reprises la production omeyyade, ce qui nous amène à élargir le champ de cette recherche à l'Antiquité tardive qui en est l'une des sources essentielles ${ }^{31}$.

La figure de la "mosquée ", dans sa conception, semble démarquée d'un modèle chrétien d'image dont la plus remarquable est une représentation de l'Anastasis, dont la rotonde est toujours mise en évidence (dans le "plan» de Madaba " ${ }^{32}$ par exemple). L'une de ces représentations, peinte à al-Bağawāt, en Égypte, dans l'Oasis de Huārğa, peut être rapprochée de notre mosaïque car la rotonde est représentée dans un contexte de colonnades à deux étages cantonnées de deux pignons ${ }^{33}$. L'architrave est interrompue par la coupole, ce qui permet de lire un développement d'une grande salle basilicale dont on a voulu représenter les supports de part et d'autre de la rotonde ${ }^{34}$.

Noël Duval propose par ailleurs une typologie où il classe dans la rubrique des « villes avec un bâtiment à coupole ${ }^{35} »$ des vignettes de l'Église Saint-Étienne d'Umm al-Rașāṣ. La bordure nilotique de celle-ci présente ainsi plusieurs villes (Panaou, Thénésos) ${ }^{36}$ dont un monument à coupole (une tholos dans les deux cas) est extrait et projeté au premier plan. Celle-ci est jouxtée de deux tours militaires qui permettent de lire la vignette comme

30. En fait, elle a été restaurée sur sa partie gauche, mais pas au centre, ce qui laisse valable le raisonnement.

31. Tous les auteurs s'accordent sur le rôle tenu par les représentations antiques dans la définition des images. De Lorey 1931 ; VAn Berchem 1969 ; Finster 1970-71; RABBAt 2003.

32. Duval, 2003, p. 218, fig. 8, b, c (n8 sur le plan) et 9a (Anastasis de Jérusalem).

33. DUVAL 2003, p. 275, fig. 28, a, b.

34. Selon un procédé de décomposition de la représentation de l'architecture bien illustré par le célèbre Psautier d'Utrecht. Voir Duval 2003, p. 216-17. Paul Lampl a également étudié cette particularité « split edifice ». Voir LAMPL 1961, p. 9.

35. Comme nous l'avons vu pour la mosquée elle-même par rapport à la ville. DuvaL 2003, p. 218, 260-266.

36. Duval 2003, p. 259-274, fig. 23, p. 262, b, c. et fig. 24, p. 263, b, c. 
une représentation symbolique de la ville par un monument caractéristique et la simple indication d'une enceinte fortifiée conventionnelle ${ }^{37}$.

Ces remarques nous permettent d'envisager que nous avons ici la représentation d'une ville par la figure du monument le plus caractéristique, ici, la « mosquée », dans un environnement urbain. Remarquons qu'il n'y a pas de fonction militaire indiquée pour ces « tours », bien que le grand appareil indiqué sur la « tour » la plus à droite y fit référence habituellement dans les modèles chrétiens.

L'aspect militaire a été rejeté hors de la mosquée dès l'époque omeyyade, il est logique qu'il le reste au moment de cette réalisation. En revanche, les tours qui se dressent derrière la mosquée constituent un rappel de la ville, très proche des figurations de certaines vignettes de l'entrecolonnement de l'Église Saint-Étienne d'Umm al-Rașāṣ, notamment celle d'Ascalon, qui comprend une rotonde et de hautes tours ${ }^{38}$.

Par ailleurs, le motif isolé (C) que l'on a proposé pour être le bayt al-māl, (et qui le représente peut-être), pourrait être lui-même extrait de la mosquée en tant que construction caractéristique, comme celle-ci l'est de la ville, pour être représenté comme un élément caractéristique du monument .

On peut penser à un raisonnement analogue pour le bâtiment (B), installé sur un podium de deux marches, dont l'aspect ouvert est celui de «l'open arcade hall » de Paul Lampl ${ }^{39}$, qui permet de montrer simultanément l'intérieur (les deux arcades) et l'extérieur, porte et chevet. Mais il est bien difficile de donner une hypothèse d'identification. Un pavillon princier dans un jardin?

Ces concepts, qui valent pour l'Antiquité tardive, jusqu'au vIII ${ }^{e}$ siècle, restent pertinents, comme l'a montré Paul Lampl ${ }^{40}$, au moins jusqu'au XII ${ }^{e}$ siècle. Nous sommes ici dans la perspective de la «longue durée », de la transmission de schémas qui servent de structure aux images tant dans la mosaïque que dans la peinture.

Le « palais » est dérivé de la formule du palais de Théodoric à Ravenne que Paul Lampl appelle « Palatium Sacrum Ravennatum » ${ }^{41}$. Ce modèle, le palais de Théodoric dans la nef de Saint-Apollinaire le Neuf à Ravenne, avait déjà été réinterprété à Damas dès l'époque omeyyade sous le portique occidental (fig. 12). L'artiste avait alors séparé le motif central, l'arc triomphal, en deux étages par une architrave qui se prolongeait en légère inclinaison sur les colonnades latérales, indiquant la manière dont doit être lue cette représentation, comme on l'a vu précédemment.

La mosaïque de la nef axiale n'adopte pas cette vision et présente une façade continue fortement suggérée, comme pour la colonnade de la mosquée, par une frise décorative évoquant des merlons, alors que la mosaïque omeyyade suggère que les colonnades latérales sont perpendiculaires à la façade ou enserrent une cour.

\footnotetext{
37. DuVAL 2003, p. 218.

38. Duval 2003 p. 247-248, fig. 19; p. 252, fig. 20.

39. LAMPL 1961, p. 11.

40. LAMPL 1961, p. 6-13.

41. LAMPL 1961, p. 12, et fig. 43.
} 
Nous retrouvons, dans notre image, la colonnade du rez-de-chaussée, mais l'arc triomphal a laissé place à un petit édicule à toit en bâtière (?) dont le pignon est marqué d'un fronton, au centre de l'étage. Le mosaïste médiéval a individualisé les étages, et réduit la place de l'élément de couronnement central en disposant cet édicule. La représentation est finalement beaucoup plus proche du modèle antique de Ravenne que du précédent omeyyade, car la frise au-dessus de l'architrave reprend l'étage de fenêtres carrées du palais de Ravenne ${ }^{42}$.

Comme à Ravenne, des coupoles occupent la partie supérieure de l'édifice, mais elles ont pris ici une place différente. Deux d'entre elles, côtelées, sont, aux angles, sur un tambour, les deux autres (en retrait?), lisses et sans tambour, portent un vase à anses à leur sommet. Ces vases sont présents dans le répertoire décoratif omeyyade ${ }^{43}$, mais il n'y a pas d'utilisation comparable de ce thème conservée dans le monument. Les vases à anses sont habituellement liés aux rinceaux et guirlandes qui se développent sur les intrados des arcs, et non à l'architecture même. Quant à ces coupoles, celles qui n'ont pas de tambour semblent disposées pour introduire une certaine profondeur dans l'image.

L'étage de l'image omeyyade (fig. 12) que nous avons rapprochée de notre mosaïque est pourvu de deux pavillons à chaque extrémité posés sur deux groupes de colonnes. C'est peut-être ce dispositif qui a été repris, en remplaçant ces pavillons par des édicules à coupoles.

Une trace (fig. 2, E et $10 \mathrm{E}$ ) du décor disparu intrigue l'observateur: un segment d'arc de couleur verte (une tige ?) conservé vient se placer là où l'on attend la troisième colonne de la partie basse du palais. Les colonnes reposent sur une base visible juste avant la grande lacune de l'image, et sont pourvues de chapiteaux. Elles sont individualisées par une forte ligne d'ombre (noire), mais nous ne pouvons pas restituer une colonnade continue de gauche à droite. Le bâtiment connaît donc un dispositif de façade qui, s'il est bien symétrique comme nous incite à le penser le traitement de l'étage, comporte au moins deux groupes de colonnes à chaque extrémité, celles-ci sont surmontées d'une coupole côtelée sur un tambour unique, et un motif central dont il ne reste qu'un très modeste vestige.

On peut risquer une hypothèse en cherchant quelques éléments de comparaison: dans le panneau de la paroi occidentale de la turba de Baybars ${ }^{44}$, le rez-de-chaussée de la composition architecturale est fait de colonnes qui portent des arcatures végétales, des rideaux courbés par le vent les obturent partiellement, par ailleurs, un écoinçon omeyyade, dans l'entrée occidentale, conserve de tels rideaux ourlés de couleur verte ${ }^{45}$. Nous pouvons imaginer un dispositif de ce type dont nous aurions là une trace, d'autant plus que la présence de rideaux dans la représentation de palais est classique.

42. LAMPL 1961, fig. 43 ; RABBAT 2003, p. 89, fig. 9.

43. Ces vases semblent dérivés, mais très simplifiés, de ceux qui ornent l'intrados d'un arc du portique ouest de la mosquée omeyyade. Voir DE LOREY 1931, pl. LXXI.

44. RABвAT 1999, fig. 6.

45. Une reproduction de cet écoinçon en noir et blanc : DE LOREY 1931, fig. 4, p. 336. 
Nous sommes bien dans le cas d'une claire filiation du type «Palatium Sacrum Ravennatum », fortement marquée par le substrat local mais sans y voir une copie servile des œuvres omeyyades. Nous avons vu que la figure doit plus à l'archétype de Ravenne qu'aux modèles (subsistants) de la mosquée même. D'autre part, les coupoles côtelées, en forme de pétales, se retrouvent, nous le verrons, très fréquemment dans la peinture de manuscrit, elles sont absentes des modèles omeyyades et ne se rencontrent guère plus dans les réalisations mameloukes où les coupoles sont pratiquement circulaires.

On peut voir à gauche, l'« ensemble F » (fig. 8), dans la partie basse de l'écoinçon, un village, un faubourg, ou peut-être un seul et même édifice en "figure désarticulée» ${ }^{46}$ en raison de la continuité des lignes entre les éléments architecturaux. L'inspiration chrétienne est très probable dans la représentation du premier corps de bâtiment couvert en bâtière et disposé en diagonale, selon une technique de perspective classique : la perspective aplanie ${ }^{47}$ qui présente de face le mur pignon et en diagonale la paroi latérale et le toit. Le second groupe architectural, ou la suite si l'on retient l'hypothèse d'un «bâtiment désarticulé » ( "split edifice »), rayé en diagonale et lié à la « tour » à coupole est représenté par le même procédé.

\section{Une relecture à l'aide de l'art byzantin médiéval est-elle possible?}

L'art byzantin, où la mosaïque tient une place éminente, peut-il être invoqué pour cette réalisation?

La représentation de l'architecture pour elle-même au xII siècle par les Byzantins est rare ${ }^{48}$, et les panneaux les plus développés sur ce thème du temps des Comnènes se trouvent dans la Basilique de la Nativité de Bethléem ${ }^{49}$, où les mosaïstes byzantins participent entre 1165 et 1169 à la réalisation d'une série de panneaux de mosaïque commandés par les Francs et dont seuls des fragments où ce thème est abordé nous sont parvenus.

Outre la représentation des villes de conciles (Nicée et Constantinople) sous la forme très simplifiée d'une double arcade frontale pour chacune, l'entrée triomphale de Jésus à Jérusalem (fig. 13) présente la ville comme une architecture fortifiée en perspective cavalière, ce qui n'est pas le cas à Damas, mais des motifs décoratifs sont parents : la coupole au-dessus du portail ressemble à celle d'un panneau dû à Nūr al-Dīn (fig. 5), mais aussi aux coupoles des groupes architecturaux de Baybars (fig. 22) ${ }^{50}$ et les fenêtres quadrilobées des écoinçons du portail, comparables à celle de la façade du groupe B (fig. 9) ${ }^{51}$.

Malgré cela, la comparaison est difficile : le byzantin se réfère aux canons de l'époque des Comnènes, où le fond or est largement dominant, l'architecture qui est sans rapport

\footnotetext{
46. « Split edifice». Voir LAmpl 1961, p. 9.

47. Duval 2003, p. 213 ; c'est la «two-sides view » de Paul Lampl. Voir Lampl 1961, p. 8.

48. Velmans 1964, p. 183-216.

49. HunT 1991, p. 69-85.

50. L'arc outrepassé est l'objet d'un modelé qui rend possible d'identifier une coupole. Le rideau qui barre la porte est un rappel du rideau du Temple. Le monument est projeté en avant de la ville car c'est son édifice emblématique.

51. Voir fenêtre du groupe B, au-dessus de la porte.
} 
d'échelle avec les personnages, est, comme l'arbre et les collines sur lesquelles courent de rares touffes d'herbes, un simple marqueur du lieu de l'action.

Au contraire, à Damas, l'architecture, est le thème central, développé sur un fond végétal très fourni, ce qui place cet ensemble en rapport étroit avec les mosaïques omeyyades malgré l'écart chronologique.

\section{La représentation de l'architecture dans les manuscrits illustrés et la mosaïque}

La peinture sur livre, à partir du début du XIII ${ }^{\mathrm{e}}$ siècle, apporte quelques représentations d'architectures qui peuvent compléter notre documentation et éclairer certains choix effectués par les concepteurs de notre mosaïque. Naturellement, la fonction de ces architectures est de servir de cadre à des scènes animées, comme nous venons de le constater pour les œuvres byzantines, ce qui n'est pas le cas dans la Mosquée des Omeyyades où elles forment le thème iconographique propre, on ne peut donc pas attendre une parfaite concordance entre les deux types de réalisations.

Asma Serajuddin ${ }^{52}$ décrit deux types de représentation de l'architecture au travers des images conçues à Bagdad entre la fin du XII ${ }^{\mathrm{e}}$ siècle et 1258. Le type I, que Richard Ettinghausen rapproche du " théâtre d'ombres " ${ }^{53}$, et le type II, beaucoup plus figuratif.

Le type I est celui d'une architecture symbolique (fig. 14) ${ }^{54}$, toujours vue de front, caractère commun avec notre mosaïque, tracée en quelques traits et matérialisée par des portes, des rideaux, quelques éléments de décor, et des coupoles formées de lobes en formes de pétales ${ }^{55}$. Ces dernières, placées au-dessus des architectures, ont pour fonction de montrer que nous sommes en présence d'une figuration en « open hall », la couverture de l'édifice étant figurée tandis que les parois sont réduites à un ensemble de lignes. Ces coupoles côtelées en pétales sont étroitement parentes de celles qui couvrent le palais aux extrémités. La grande coupole de la « mosquée » est elle aussi composée dans cet esprit.

Le type II est caractérisé par un plus grand « réalisme » : les fonctions des lieux sont identifiables (mosquées, palais, caravansérails, paysages...). On y trouve des notations parfois très précises comme, par exemple, l'inscription sur une mosquée qui a permis de dater le manuscrit d'Istanbul ${ }^{56}$. C'est cependant la représentation de l'espace qui y est le caractère le plus original, notamment par la mise en place de plans successifs et de stratagèmes pour susciter l'idée de profondeur.

52. SERAJUDDIN 1975 , p. 31-40.

53. ETTINGHAUSEN 1977, p. 105. Ces décors empruntent beaucoup à l'aspect des ornements mobiliers en bois connus pour les XII et XIII ${ }^{e}$ siècles (maqșūra de la Mosquée Muṣallā l-'īldayn à Damas, minbar-s, poutres à décor de polygones assemblés, décor en «taille oblique », etc. dont les exemples sont nombreux).

54. Il faut y voir des architectures tripartites comportant souvent une sorte d'īwān central cantonné de deux petites salles latérales.

55. Voir illustrations : ETTINGHAUSEN 1977, p. 106-107, 108 et 113.

56. JAMEs 1974, p. 306. La copie d'Istanbul n'est pas datée, mais contient une inscription au nom du calife alMusta'șim (640-656/1242-58). 
La représentation de l'espace intérieur et extérieur simultanés reste la règle ${ }^{57}$. Une mosquée (fig. 16), dans le manuscrit d'Istanbul ${ }^{58}$, présente une scène où l'architecture est ouverte tout en conservant des détails caractéristiques de l'extérieur ${ }^{59}$.

Les deux arcades de façade portent une corniche sur laquelle se développe un entrelacs très complexe qui se rattache aux jeux de briques (hezārbā ${ }^{60}$ ) iraniens et mésopotamiens, mais qui comprend également un lacis de figures géométriques qui se termine en une ligne de bobéchons entre deux bornes faîtières, porteuses elles aussi d'un entrelacs géométrique. D'autres mosquées de ce type (fig. 17) comportent un niveau de merlons découpés. On y sent ici l'influence de l'architecture, mais aussi des décors des arts de la métallurgie que suggèrent les couleurs choisies (rouge, jaune).

La salle de prière, hypostyle, est présentée, pour la première mosquée, à travers deux arcades, trois pour la seconde, dont une colonne est placée devant le mihrāb, ce qui induit un sentiment de profondeur. Le minbar est adossé au mur droit dans l'une, gauche dans l'autre, est présenté de profil, ce qui permet d'identifier immédiatement ce meuble très caractéristique. Le mobilier de la mosquée est complété par les lampes suspendues. Tout cela est très classique et relève de la convention.

La richesse de la représentation est évidemment plus grande que celle de la mosaïque qui nous préoccupe, toutefois la mosquée est bien présentée à la fois intérieurement et extérieurement dans les deux cas, et on trouve, dans la grande arcade centrale de la mosaïque, avec la figuration décalée d'une seconde arcade, un procédé de traduction de la profondeur comparable à la multiplication des plans dans les peintures.

Pour mémoire enfin, citons le folio 138 (recto) du manuscrit Schefer ${ }^{61}$ qui présente la rencontre d'Abū Zayd, devant une ville, avec deux interlocuteurs montés sur des dromadaires (fig. 15). La ville, au-dessus, est comme posée sur un sol figuré en arc de cercle.

La ville est elle-même composée de deux autres plans : le premier, où l'on pénètre par une porte fortifiée, est un alignement d'échoppes étroites et verticales, ouvertes chacune par un arc, et dont la couverture est une voûte de pierre (ou une coupole?).

Le second plan, celui qui nous intéresse, est celui de la mosquée, exceptionnellement vue totalement de l'extérieur, partiellement cachée par ces boutiques pour augmenter la dimension de la profondeur. Elle est pourvue d'un minaret dont le fût circulaire a reçu, comme le mur de clôture, une grande inscription coufique qui fait référence aux

57. BARRUCAND 1994, p. 80 ; LAMPL 1961, p. 9.

58. Grabar 1970, fig. 3 (p. 104 du manuscrit d'Istanbul). La même scène apparaît dans le manuscrit Schefer, voir Maqāmāt d'al-Ḥarīrī, Manuscrit arabe 5847, peint par Yahyā b. Maḥmūd al-Wașītī, Bibliothèque nationale de France, folio $164 \mathrm{v}$.

59. LAMPL 1961, p. 11. Type: « Opening of the Wall and Exposure of the Interior ».

60. Décors de jeux de briques très prisés dans la période seldjoukide.

61. EtTINGHAUSEn 1977, p. 116 (Bnf. Arabe 5847, [manuscrit Schefer], folio 138 recto). 
caractéristiques des mosquées seldjoukides du XII ${ }^{\mathrm{e}}$ siècle ${ }^{62}$ et surtout une vaste coupole ${ }^{63}$, conçue comme celles des mosquées iraniennes.

Cette représentation complexe, bien éloignée de notre mosaïque montre toutefois l'une des seules grandes coupoles représentées en enluminure, sans que l'on puisse trancher sur une question: s'agit-il d'une coupole côtelée ou de l'indication du tracé des feuilles de plomb qui la couvrent ? ${ }^{64}$ On rencontre sur ce document une certaine variété de la figuration des matériaux que les mosaïstes de Damas ont, eux aussi cherché à restituer, la pierre de taille (la porte de la ville a un appareil à bossage), la brique (boutiques), le bois (balconnet du minaret).

La profondeur est rendue de deux façons: la multiplication des plans, mais aussi l'ellipse (ou la colline) qui constitue la base de la scène, ce que l'on retrouve à la fin du XIII ${ }^{\mathrm{e}}$ siècle dans la figuration des architectures des mosaïques du mur nord (fig. 22) de la turba de Baybars ${ }^{65}$.

Le palais, lorsqu'il est représenté, est soit une structure fermée, sur deux étages (type II), vue de l'extérieur, en vision frontale, image qui, selon Asma Serajuddine, ${ }^{66}$ est ancrée dans la culture persane, soit il appartient au type I.

Les solutions retenues pour le traitement de l'espace dans les manuscrits sont donc nombreuses, mais la représentation frontale demeure la règle principale. La multiplication des plans et le chevauchement des figures, la dislocation des espaces dans certains cas (nous l'avons vu dans le type I) sont les moyens de traduire la profondeur.

Le livre, donc, s'il est un vecteur de diffusion des images ${ }^{67}$, semble avoir assez peu contribué à la formation des images de la nef axiale de la Grande Mosquée de Damas. Il semble donc que le répertoire des mosaïstes était fondé sur une tradition plus ancienne et des cartons dont nous avons vu qu'ils comportaient d'évidents archaïsmes.

\section{À quels moments aurait pu se situer la réalisation de cette mosaïque ?}

Marguerite Van Berchem ${ }^{68}$, complétant son enquête à l'aide du croquis réalisé par Michel Écochard (fig. 2) lors de travaux de restauration qui lui avaient permis une observation assez précise de l'ensemble supérieur ${ }^{69}$, propose d'attribuer ce panneau, en invoquant des arguments stylistiques, aux grands travaux de restauration de 475/1082,

62. Les minarets de l'époque seldjoukide sont porteurs de grandes inscriptions en caractères coufiques développées en bandeaux. Voir BLoom 1989, p. 157-174 (nombreux exemples illustrés).

63. La salle à coupole est un élément essentiel de l'architecture seldjoukide (Isfahan, etc.), la représentation de ce manuscrit est difficile à interpréter : s'agit-il d'une coupole côtelée ou de la représentation des nervures qui résultent de la couverture externe en plomb d'une coupole lisse?

64. La couleur suggère cet élément dont on sait qu'il couvrait les toitures et la coupole de la Mosquée de Damas.

65. RABBAT 1999, fig. 7, 8.

66. SERAJUDDINE 1975, p. 37, et fig. 8.

67. Ali 2007, p. 111, qui reprend l'hypothèse de catalogues circulant entre les mains des artistes.

68. VAN BERCHEM 1969, p. 323-372.

69. VAN BERCHEM 1969, p. 355. Ce croquis date de 1937. Il me semble toutefois que ce croquis est davantage une interprétation qu'un relevé. Je l'ai complété à l'aide d'observations faites sur site, mais hors échelle. 
menés par Abū Nașr Aḥmad, l'un des vizirs de Tutuš, le frère de Malikšāh en charge de Damas $^{70}$.

Les éléments du décor attribués à l'époque seldjoukide (décor en zigzags ou «bâtons rompus », entrelacs géométrique, etc.) et l'idée que la restauration de Malikšāh est très importante ${ }^{71}$ sont les arguments essentiels de Marguerite Van Berchem. Ces travaux conséquents et bien attestés ${ }^{72}$ auraient pu être l'occasion de reprendre le décor intérieur de ce pignon, mais aucun document ne les mentionne ${ }^{73}$.

D'autres pistes, dans la mosquée même, permettent de suggérer d'autres moments :

1. L'angle nord-est de la cour conserve une inscription de deux lignes sans date, incomplète, en nashīnoir sur fond or, en mosaïque de pâte de verre (fig. 4). Si on ignore ce que commémore cette inscription de deux lignes, la seconde ligne mentionne «al-Malik al-'Ádil al-Zāhid al-Murābiț al-Muğāhid Nūr al-Dīn Abū l-Qāsim Mahmūd», qui permet l'identification certaine de Nūr al-Dīn ${ }^{74}$, dont l'activité à Damas, se situe entre 1154 (entrée du prince dans la ville) et 1171 (date de son décès).

Non loin de cette inscription, le décor de la partie supérieure du pilier du portique oriental le plus proche de l'angle nord-est (fig. 5) comprend une seconde inscription en mosaïque, en cubes noirs sur fond or que Gaston Wiet ${ }^{75}$ propose de dater du début de l'époque ayyoubide ${ }^{76}$ ou plus précisément de celle de Nūr al-Dīn.

2. Des inscriptions sur marbre ${ }^{77}$ commémorent d'autres travaux sous le règne de Saladin dans la nef axiale de la Grande Mosquée : il s'agit de la restauration du placage des piliers de la salle de prière, mais rien n'est dit sur une éventuelle mosaïque ${ }^{78}$.

70. VAN BERCHEM 1969, p. 354.

71. L'architecte de Tutuš entreprend la reconstruction de la coupole sur une nouvelle échelle, entraînant un remaniement global de la nef axiale, et notamment de ses supports. Les modestes vestiges des mosaïques des piliers de la coupole n'ont pas le même caractère que celle qui nous préoccupe et datent vraisemblablement de l'époque de Malikšāh. Voir VAN BERCHEM 1969, p. 323-372.

72. De grandes plaques de marbre conservées au Musée national de Damas portent des inscriptions qui les commémorent. RCEA VII, n²734 - 2736, 3737 (475 H/1082 AD) ; AL-'UsH 1976, p. 254, fig. 145.

73. Nasser Rabbat a montré que si les mosaïques de la Grande Mosquée sont évoquées dans les sources historiques, ces textes sont centrés sur l'abondance végétale représentée, mais ne mentionnent pas l'architecture, qui a pourtant une place essentielle dans l'œuvre omeyyade. Ce panneau tardif ne l'est pas davantage. RABBAT 1999, p. 12 ; RABBAT 2003, p. 91.

74. ÉLISSÉEFF 1949-51, n³9, p. 22 ; ÉLISSÉEFF 1952-54, p. 159, nVI. L'auteur la date grâce aux textes historiques (554/1159-60), [localisation erronée : confusion est-ouest] ; VAN BERCHEM 1969, p. 352, et fig. 413.

75. VAN BERCHEM 1969, p. 350-351 et fig. 410. La lecture publiée est de Solange Ory et Marguerite Van Berchem s'appuie sur l'autorité de Gaston Wiet pour dater ce panneau du règne de Nūr al-Dīn. Le caractère proche de l'écriture nashī employée de même manière dans ce document et le précédent incline à le penser.

76. Le mot est utilisé ici dans un sens large, comprenant la période de l'arrivée des Seldjoukides à l'avènement des Mamelouks.

77. Elles sont conservées au Musée national de Damas. Elles concernent le revêtement de marbre de piliers, mais n'évoquent pas d'autres travaux d'embellissement. Voir AL-'UsH 1976, p. 254. RCEA, VII, n³343 et 3344.

78. Rappelons cependant que le règne de Saladin a ramené la Palestine dans l'orbite islamique où une certaine permanence de l'art de la mosaïque est attestée dans l'espace ayyoubide (conquête de Jérusalem en 1187). Cf. RosENAYALON 2002, p. 106 et $131-133$. 
3. Une série de grands travaux a lieu au temps d'al-'Ádil, comme le pavage de la cour (achevé en 1208) pour lequel il fait démonter des arcades près de Bāb al-Šarqī pour obtenir les matériaux nécessaires, ou le dallage de la salle de prière commencé en 1214 et achevé en 1217, ainsi que des travaux de charpente à la coupole (1216) ${ }^{79}$. Ces travaux, marquant une volonté d'embellissement, sont une occasion vraisemblable, mais non documentée.

4. Baybars ${ }^{80}$, fait restaurer des mosaïques de la Grande Mosquée. Il a laissé à cette occasion une grande inscription dans le vestibule ouest de la Grande Mosquée, Bāb alBarīd. On croit reconnaître ces travaux sous le portique ouest, à proximité de l'angle nord-ouest ${ }^{81}$, mais leur style et leur schéma structurel, comme celui des mosaïques qui ornent son mausolée, qui datent du règne de Qalāwūn ${ }^{82}$, diffèrent beaucoup des images qui nous préoccupent.

\section{Confrontation et contexte du mécénat}

Nous avons vu combien la représentation tant de la mosquée que du palais dépend des images de l'Antiquité tardive plus encore que de la période omeyyade. On a l'impression qu'il s'agit de vignettes que l'on a puisées dans le répertoire chrétien, mais adapté à leur nouvelle fonction.

Le traitement du bâtiment (B), ouvert d'une porte très verticale avec un arc en plein cintre, suivie de deux arcades et fermé par une "tour » à décor d'entrelacs présente bien le problème. On peut y voir une salle basilicale, " architecture ouverte » ${ }^{83}$, dont l'abside, courante en milieu chrétien, est remplacée par l'entrelacs. L'élévation intérieure est vue sous la forme de deux arcades où des fleurons ont pris place. L'entrelacs et les fleurons islamisent le motif, qui semble avoir été placé là pour des raisons structurelles d'équilibre de la composition, beaucoup plus que pour une signification propre qui reste énigmatique.

L'inspiration directe de modèles anciens, peut-être chrétiens, me semble possible, car le recensement des églises de Damas par Ibn 'Asākir ${ }^{84}$ rappelle que des modèles peuvent exister jusqu'en ville. Nous avons souligné certaines maladresses qui incitent à voir l'adoption d'images tirées d'un «catalogue » ou d'un modèle : l'ensemble A (la " mosquée ») est comme "rogné » par la courbe de l'arcature que le panneau encadre, chacune des architectures de la partie haute est individualisée et comme posée sur une ligne horizontale, comme les vignettes individuelles que l'on trouve dans les églises byzantines.

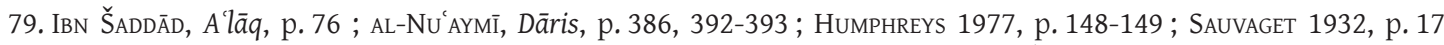
(chronologie sans appareil critique des travaux dans la Grande Mosquée : p. 15 à 18).

80. SAUVAiRe 1894-96, p. 282 ; VAN BerChem 1969, p. 331, fig. 388.

81. VAN BERCHEM 1969, fig. 406- 407.

82. Flood 1997, p. 60-61; RABBAT 1999, p. 3-6. Ces deux auteurs suivent Meinecke.

83. LAMPL 1961, p. 9-10.

84. ÉLISSÉEFF 1959, p. 214-223. Certaines d'entre elles sont en ruine à son époque. Beaucoup ont pu comporter de telles vignettes. 
Aucune des pages de manuscrits que nous avons étudiées ne comporte une mosquée d'aspect comparable à celle de la mosaïque. Mais, à une exception près, ce type de monument est toujours figuré de manière frontale, en structure ouverte avec deux ou trois arcades où pendent des lampes, et en une image qui présente tout à la fois l'intérieur (la salle de prière) et l'extérieur (couronnement de la façade, minaret). La profondeur est toujours suggérée par des superpositions de plans ou d'objets. Ce qui n'est pas sans rapport avec la représentation de la façade dont l'espace est approfondi et suggéré par le décalage d'une seconde arcade, qui comporte en outre un couronnement de motifs en « T » tête-bêche qui rappellent bien le hezārbāf que nous avons évoqué dans le type II.

Malgré cette parenté, la part de l'Antiquité tardive est déterminante, plus encore que celle des panneaux omeyyades. C'est la tradition la plus ancienne qui l'emporte pour la représentation du palais, moins exubérante que celles du viII ${ }^{\mathrm{e}}$ siècle qui s'attache plutôt du scenae frons, le mur de scène du théâtre romain, que de l'architecture aulique. Malgré la présence importante de la végétation, notamment dans les parties excentrées de la composition, mais aussi comme moyen de liaison retenu par les artisans, le contexte omeyyade s'efface devant un archaïsme plus puissant.

La représentation d'architectures selon les modes que nous avons décrits fait appel à une technique qui s'appuie sur la longue durée, depuis l' «Ecclesia Mater » de Tabarka (fin $\mathrm{IV}^{\mathrm{e}}$-début $\mathrm{V}^{\mathrm{e}}$ siècle) jusqu'à la fin du Moyen Âge, la conception d'un édifice présenté à la fois extérieurement et intérieurement par la décomposition de ses éléments, la disparition de certaines parois et l'aplanissement de la figure. Noël Duval ${ }^{85}$ précise d'ailleurs que le type iconographique de la basilique, dans le Psautier d'Utrecht (début IX siècle), s'accorde aussi bien pour représenter un palais qu'une église.

Cet archaïsme n'est pas le fruit du hasard, il faut alors le rapprocher de moments précis de la création artistique en Syrie, et plus particulièrement à Damas.

Outre les travaux réalisés au nom de Malikšāh, le mécénat seldjoukide connu à Damas se résume à un monument énigmatique de Tutuš (fin $\mathrm{xI}^{\mathrm{e}}$ siècle) connu seulement par des inscriptions coufiques réalisées sur des tables de marbre byzantines en remploi dans un monument du XVI ${ }^{\mathrm{e}}$ siècle ${ }^{86}$, et à un autre monument, disparu en 1938 , le tombeau de Safwat al-Mulk ${ }^{87}$. Il est difficile de se faire une opinion sur la conception qu'auraient eue ces princes de l'éventuelle réalisation d'une image, mais la construction de la coupole révèle un goût nouveau pour la monumentalité de l'architecture. La grande coupole est construite en bois mais avec des dimensions qui la rapprochent des coupoles iraniennes. Il n'est pas certain que cette innovation s'accorde avec le côté très conservateur des images que nous étudions.

85. DuVAL 2003, p. 213-214, fig. 1-3 a-b.

86. Elles ont été remployées dès le XII ${ }^{\mathrm{e}}$ siècle dans un autre monument, puis une seconde fois au XVI ${ }^{\mathrm{e}}$ siècle vers la Madrasa al-Sibāi iyya où elles sont encore en place SoURdeL-THomine 1979, p. 144-146, pl. 8-9 ; FLood 2003, p. 145-154. 87. SAUVAGET 1938. 
Le règne de Nūr al-Dīn, particulièrement fécond en production artistique, présente des caractères qui étaient apparus à Terry Allen comme ceux d'une « Renaissance classique » ${ }^{88}$. Il s'est attelé, à partir d'un monument alépin, le Qasțal al-Šu'aybiyya, dont la structure lui paraissait calquée sur les schémas antiques, à définir un courant puisant largement dans le répertoire architectural classique au milieu du XII ${ }^{\mathrm{e}}$ siècle. Yasser Tabbaa ${ }^{89}$ et Julian Raby ${ }^{90}$ ont depuis nuancé cette vision en mettant en évidence une continuité stylistique dans un contexte monumental riche en éléments antiques. Il reste toutefois que nous pouvons remarquer plusieurs traits de la démarche visible dans le mécénat de Nūr al-Dīn. En effet, il importe des formules architecturales clairement mésopotamiennes avec les coupoles à muqarnas en pain de sucre et fait venir des constructeurs d'Alep dont on reconnaît la main dans les procédés de construction. Mais surtout, dans deux monuments conservés de Damas ${ }^{91}$, il fait usage de spolia antiques d'une manière qui semble délibérée.

Le premier monument est le bìmāristān qu'il fait édifier intra muros, où est inséré, sous une demi-coupole à muqarnas en stuc et au-dessus du portail rectangulaire, un fronton antique placé là avec son fin décor de rinceau et ses moulurations en fort relief ${ }^{92}$. Dans le même monument, il a réuni une collection de tables de marbre blanc ${ }^{93}$ en forme de sigma pour les unes, rectangulaires avec un décor lobé pour les autres (fig. 19). Toutes proviennent d'églises byzantines. Sur l'une de ces tables, dans l'īwān oriental, est creusée l'inscription emplie de pâte noire qui identifie le fondateur. La raison de cette collection n'est pas claire, mais il y a une admiration pour le matériau noble qu'est le marbre blanc, « marbre royal, al-ruham al-malikī» dont Finbarr B. Flood souligne l'intérêt, avec la technique de l'incrustation, de la lisibilité des inscriptions ${ }^{94}$.

Le second monument est le dār al-hadiț ${ }^{95}$ qu'il a fait édifier près de la porte orientale de la citadelle. Là, le portail reprend, par ses moulures le schéma décoratif d'un portail antique ${ }^{96}$, mais surtout la conque bordée de guirlandes du mihrāb est une pièce antique en remploi (fig. 20), dont la destination a été islamisée.

L'inscription en mosaïque au nom de Nūr al-Dīn en nash̄ī, graphie cursive dont Nūr alDīn généralise l'emploi dans les monuments qu'il fonde ${ }^{97}$, est réalisée avec grand soin,

\section{ALLEN 1986.}

89. TABBAA 1993.

90. RABY 2004. J. Raby convient tout de même que le qasțal est bien conçu en référence au passé, mais par référence au début de l'islam, et non à l'Antiquité gréco-romaine.

91. On devrait ajouter encore la table d'autel qui avait été insérée dans le mur de la turba de sa madrasa funéraire. Celle-ci a été photographiée par Creswell, mais a disparu depuis. Voir FLood 2001, p. 56, fig. 22.

92. J. Raby l'interprète comme un rappel du fronton caractéristique de la nef axiale de la Grande Mosquée, et comme un élément caractéristique de la Syrie que Nūr al-Dīn vient d'unifier en entrant à Damas où ce monument est le premier qu'il construit dans la ville. Voir RABY 2004, p. 305.

93. La fonction de ces tables n'est pas vraiment claire, l'expression « table d'autel » leur est couramment attribuée. Voir Flood 2001, p. 57.

94. FLOOD 2001, p. 53.

95. SAUVAGET 1938, p. 15-25.

96. Une porte de ce type a été remployée dans la mosquée d'Urfa à la même époque. GuIDETTI 2009, p. 18, fig. 12.

97. TABBAA 2001, p. 53-72. Le nash̆i est employé pour la première fois pour une inscription monumentale à Damas dans l'inscription de fondation de la Madrasa al-Muǧāhidiyya (539/1144). 
tout comme l'autre inscription, elle aussi en nashī, sur le portique nord, mais à immédiate proximité du même angle, qui lui est attribuée avec vraisemblance en raison de la grande parenté des caractères. Ces textes, surtout le second, sont insérés dans un ensemble qui n'appartient pas à l'époque omeyyade, où l'on distingue des éléments décoratifs originaux.

Une coupe à pied (fig. 4) d'où sortent des végétaux dérivés de l'acanthe fait écho aux décors végétaux des écoinçons et des intrados des arcatures omeyyades sous l'inscription de Nūr al-Dīn, celle-ci a un encadrement fait d'un réseau de brins entrelacés de couleur verte sur fond or.

Le curieux cadre de la seconde inscription, composé de deux colonnes portant une architrave parcourue d'un motif ondulé, est un élément de comparaison important. Ce dernier semble être le souvenir d'un dispositif antique de coupoles qui couronnait la figure des mois dans les calendriers antiques ${ }^{98}$. Enfin, une coupole en demi-cercle outrepassé, composée de motifs concentriques allant du bleu au rouge en passant par le jaune ${ }^{99}$, est posée sur un motif ondulé ${ }^{100}$, et prend place au-dessus de l'inscription elle-même, entre les deux colonnes.

$\mathrm{Au}$-dessus de cette inscription se développent, difficilement lisibles mais bien présents, des fragments de décors architecturaux formés de chevrons affrontés (fig. 6), tout à fait comparables à ceux que l'on trouve sur la « hazna» (fig. 10, C). de la mosaïque de la nef axiale. On le retrouve également sur une peinture de la même époque (milieu XII ${ }^{\mathrm{e}}$ siècle), au plafond de la chapelle palatine de Palerme, sur la représentation d'une fontaine ${ }^{101}$ où chaque ligne des bâtons brisés est peinte de couleur différente. Il est difficile de trouver une interprétation convaincante à ce motif, mais il est clairement lié au milieu du XII siècle, et à Damas, probablement à Nūr al-Dīn ${ }^{102}$.

Un autre élément peut être situé dans le temps : l'entrelacs rubané vertical fermé en forme de nœud de l'une des vignettes (fig. 4,B). Les exemples d'entrelacs géométriques sont assez nombreux aux XII ${ }^{\mathrm{e}}$ et $\mathrm{XIII}^{\mathrm{e}}$ siècles en Syrie à Alep notamment ${ }^{103}$. Ce type d'ornement venu d'Orient s'affirme dès la fin du XI $\mathrm{x}^{\mathrm{e}}$ siècle ${ }^{104}$ et Yasser Tabbaa ${ }^{105}$ estime que le mécénat de Nūr al-Dīn fut déterminant pour son introduction en Syrie, ce qui constitue un premier jalon pour notre projet de datation : ce prince n'est entré à Damas qu'en 1154 et y mourut

98. HoffmAn 2000, p. 37-52. Voir Calendrier de 354, fig. 8, 9, 10, p. 43-44. L'auteur établit une filiation probable entre art romain tardif et peinture fatimide, mais s'intéresse à la figure humaine plus qu'au cadre architectural.

99. On trouve de telles coupoles dans les mosaïques omeyyades, en couronnement de certaines tours. Mais elles sont toujours de petites dimensions. Voir DE LOREY 1931, pl. LXXII.

100. Peut-être là aussi un rappel du motif qui couronne le panneau et évoque les coupoles antiques?

101. Ettinghausen 1977, p. 48. Il s'agit d'une fontaine, visible d'ailleurs au palais de la Ziza, avec un décor de chevrons en relief.

102. Nūr al-Dīn et son entourage ont eu une grande activité architecturale en Ǧazīra, région de l'architecture de briques. Voir HiLlenBRAND 1985, p. 21-48.

103. Par exemple, le Mašhad al-Husayn (entre 569/1176 et 596/1199) : encadrement de l'īwān et miḥrāb. Voir TABBAA 2001, p. 158 (ill. 83) et 159 (ill. 84) ; la Madrasa al-Šādbahtiyya, miḥrāb avec cadre en entrelacs, (589 ou 599/ fin XII ${ }^{\mathrm{e}}$ siècle. Voir HERZFELD 1953-1955, p. 255.

104. L'ensemble des palais de Lashkari Bazar en comporte de beaux exemples. TABBAA 2001, p. 78, fig. 28.

105. TABBAA 2001, p. 73-102 (notamment p. 100). 
en 1171. Cependant, on retrouve ce motif pendant la période mamelouke ${ }^{106}$. À Damas une pierre en remploi, non datée (fig. 18) dans le mur Nord de la citadelle montre des caractères très proches ${ }^{107}$.

Il est difficile d'évoquer autrement que comme dates possibles les règnes de Saladin et d'al-'Ádil : le mécénat du premier à Damas est modeste, mais il est intervenu dans la restauration des placages de la nef axiale. Quant au second, il a fait de nombreux travaux dans la Grande Mosquée, mais il semble que ces derniers fussent plus particulièrement d'ordre architectural.

Seul Baybars pourrait apparaître comme un autre candidat sérieux pour l'exécution de ces mosaïques de la nef axiale. Nous disposons d'un panneau qui lui est attribué dans la mosquée, et de ceux qui ont été exécutés par Qalāwūn dans sa turba.

La comparaison favorise cependant l'hypothèse $d u$ XII $^{\mathrm{e}}$ siècle : la représentation des coupoles en pétales de notre panneau ne coïncide pas avec les bulbes presque circulaires de la turba ; la conception du panneau de la qibla de cette dernière, en deux étages, rappelle le scenae frons cher aux Omeyyades, mais ce dispositif est absent du panneau de la nef axiale, où l'idée d'un second étage est même gommée dans la représentation du palais, où l'on a figuré des fenêtres plutôt que des colonnades; enfin, la perspective des images mameloukes (fig. 22) reprend d'une certaine façon l'ellipse que nous avons trouvée dans les manuscrits des Maqāmāt d'al-Harīīi (fig. 15), ce que nous ne trouvons pas dans le panneau médiéval de la Mosquée des Omeyyades où la seule représentation de la profondeur se fait par des plans superposés ou des lignes diagonales. En somme, l'art de la mosaïque, chez les mécènes mamelouks s'inspire très étroitement, et délibérément ${ }^{108}$, des réalisations omeyyades en y associant des procédés issus des images de la peinture sur manuscrits, alors que la mosaïque que nous cherchons à dater s'inspire beaucoup plus directement de l'art chrétien de l'Antiquité tardive, d'une façon d'ailleurs étonnante qui n'est tempérée que par la place accordée à la végétation représentée.

\section{Conclusion}

La discussion qui précède et l'épigraphie du monument lui-même invitent à penser que Nūr al-Dīn est le commanditaire le plus probable : son mécénat, à Damas, mais aussi dans tout l'espace politique qu'il contrôle, notamment en Syrie du Nord, comporte un nombre important de remplois, ou de citations du répertoire du passé.

Le sens de cet usage rappelle l'impact qu'avait eu sur les conquérants les monuments chrétiens aux débuts de l'islam : au viII ${ }^{\mathrm{e}}$ siècle, époque où le christianisme reste la religion dominante, la Cathédrale Sainte-Sophie de Ruah est, pour al-Muqaddasī (966-1000) une

106. Encadrement du portail de la Turba al-Silaḥdār dans le quartier du Mīdān, par exemple.

107. Signalons aussi une œuvre d'époque ayyoubide, le mihrāb «passementé » de la turba de Maḥmūd b. Zankī (625/1227-28). Voir SAUVAGET 1932, p. 64, n³4.

108. FLOOD 1997. 
merveille égale à la Mosquée al-Aqșā de Jérusalem et à la Mosquée de Damas ${ }^{109}$. Les mosaïques de Damas et de Jérusalem devaient donc égaler ou surpasser le modèle chrétien. Au XII ${ }^{e}$ siècle, la plupart des églises tombent en ruines, certaines sont converties en mosquées, en madrasas ${ }^{110}$ ou deviennent des dépôts de matériaux que l'on remploie ${ }^{111}$. Cette perspective est très différente de celle du premier siècle de l'islam, et le sens que l'on peut donner à la citation de l'antique n'est plus le même.

La destination iconographique propre nous échappe, comme l'a démontré Nasser Rabbat, faute d'écrits sur les architectures avant le xiv siècle dans les descriptions médiévales ${ }^{112}$. L'iconographie des panneaux omeyyades a été l'objet d'études dont celle de Barbara Finster ${ }^{113}$ qui a montré que des textes coraniques de nature eschatologique figuraient dans la salle de prière; elle les a mis en relation avec les images produites, donnant ainsi une idée paradisiaque de l'ensemble. Nasser Rabbat a donné une nouvelle lecture à cet ensemble où il voit plutôt une représentation du monde, sur le modèle des mosaïques chrétiennes comme celle de la «carte» de Madaba ${ }^{114}$. Mais il est difficile de savoir, en ce qui concerne le panneau que nous avons étudié s'il est à mettre en relation avec l'iconographie omeyyade ou s'il faut y voir une nouvelle signification ${ }^{115}$.

La réalisation des images de la nef axiale, quel que soit le propos iconographique, se place à un moment où l'usage des remplois dans l'architecture est très fréquent, dans la seconde moitié $\mathrm{du} \mathrm{xI}$ siècle. Nous avons mentionné quelques exemples damascains, mais ce courant affecte avant tout la Syrie du Nord où le mécénat zankide ${ }^{116}$ s'exerce avant l'entrée à Damas et où la tradition byzantine est très prégnante.

Ces citations antiques, sans lien avec le contexte initial, apparaissent comme un usage purement formel de l'image, ce qui diffère profondément de l'intention de Baybars et Qalāwūn, qui, eux se réfèrent clairement à la période omeyyade, des débuts de l'islam ${ }^{117}$. De telles dispositions se retrouvent, par exemple, dans la porte de la forteresse de Harrān, où des sculptures antiques sont remployées pour orner les écoinçons ${ }^{118}$.

\footnotetext{
109. GUIDETTI 2009, p. 8.

110. Madrasa al-Hallawiyya à Alep, par exemple.

111. GUIDETTI 2009, p. 22.

112. RABBAT 1999, p. 13.

113. FinSTER 1971, p. 118-121; RABBAT 2004, p. 89.

114. RABBAT 2003, p. 92-93.

115. Remarquons toutefois que nous connaissons le thème qui faisait face à ce panneau : une représentation de la Ka'ba. Si la datation proposée est correcte, compte-tenu de la personnalité de Nūr al-Dīn, le monument à coupole pourrait-il être une évocation de la Mosquée al-Aqșā ? La prise de Jérusalem est l'objectif avoué du prince. Mais cette identification se heurte à l'interprétation difficile des autres éléments représentés.
}

116. Allen 1986 ; TABBAA 1993 ; RABY 2004.

117. FLOOD 1997, p. 57-79.

118. AlLEN 1986, fig. 46.-48. 


\section{Bibliographie}

\section{Sources}

IBN ŠADDĀD, A 'āq al- hatịira fi dịkr umarā' al-Šām wa-l-Ğazīra : tārīh Dimašq, la description de Damas d'Ibn Šaddād, Historien et géographe mort à Alep en 684/1285, éd. Sami Dahan, Damas, Institut français de Damas, 1956. Nu'AYMī (AL-), al-Dāris fì tārīh al-madāris, 2 vol., éd. Ǧa far al-Ḥasanī, Damas, 1948-1951.

\section{Études}

ABu'L-FARAĞ AL-'Ush, Muhammad, Joundi, Adnan, Zouhdi, Bachir, 1976 : Catalogue du musée national de Damas, Damas.

ALLEN, Terry, 1986: A classical Revival in Islamic Architecture, Wiesbaden.

AlLen 2003 : Ayyūbid Architecture, Occidental (publication électronique), $7^{7}$ édition.

Ali, Nadia, 2007 : «Qusayr Amra, la peinture du personnage trônant sur l'eau : aspects pratiques de la fabrication de l'image », Annales islamologiques 44, p. 107-137.

ATIL, Esin, 1975: Art of the Arab World, catalogue de la Freer Gallery of Art, Smithsonian Institute, Washington.

BARRUCAND, Marianne, 1994 : «Architecture et espaces architecturés dans les illustrations des Maqāmāt d'al-Harīrī », in Robert HillenBRAnd (éd.), The Art of the Saljûqs, Costa Mesa, California, p. 79-88.

BLoom, Jonathan, 1989 : « The Saljuq Minaret, », in Minaret, Symbol of Islam, Oxford studies in Islamic art, vII, Oxford.

Combes, Etienne, SAUVAGET, Jean, Wiet, Gaston et alii, 1937-ss : Répertoire Chronologique d'Epigraphie Arabe (RCEA), vol. vII et VIII, Le Caire.

CRESWELL, K. A. C., 1969 : Early Muslim Architecture, II, Oxford, $2^{e}$ édition.

DE LOREY, Eustache, 1931 : « Les mosaïques de la mosquée des Omeyyades à Damas », Syria 12, p. 326-349.

DuvAL, Noël, 2003 : «Les représentations architecturales sur les mosaïques chrétiennes de Jordanie », in Noël Duval \& Ernest Will (éd.), Les églises de Jordanie et leurs mosaïques, Lyon, p. 211-285.

ÉLISSÉEFF, Nikita, 1949-51 : «Les monuments de Nūr al-Dīn », Bulletin d'études orientales 13, p. 5-43.

ÉLISSÉEFF, Nikita, 1952-54 : « La titulature de Nûr al-Dîn d'après ses inscriptions », Bulletin d'études orientales 14 , p. 157-196.

ÉLISSÉEFF, Nikita, 1959 : La description de Damas d'Ibn Asākir, Historien mort à Damas en 571 H/1176, Damas, Institut français de Damas.

HUMPHREYS, R. Stephen, 1977 : From Saladin to the Mongols, New York/Albany.

EtTinghausen, Richard, 1977 : La peinture arabe, Genève. 
Finster, Barbara, 1970-71 : «Die Mosaiken der umayyadenmoschee von Damaskus », Kunst des Orients 7/2, p. 83-136.

Flood, Finbarr B., 2001: «A group of Reused Byzantine Tables as evidence for Seljuq Architectural Patronage in Damascus », Iran 39, p. 145-154.

Flood, Finbarr B., 2001: « The Medieval Trophy as an Art Historical Trope: Coptic and Byzantine "Altars " in Islamic Contexts », Muqarnas 18, p. 41-72.

FLood, Finbarr B., 1997 : «Umayyad Survivals and Mamluk Revivals : Qalawunid Architecture and the Great Mosque of Damascus », Muqarnas 14, p. 57-79.

GRABAR, André, 1994: Les voies de la création en iconographie chrétienne, Paris (Première édition, 1979, réédition Flammarion, 1994).

GRABAR, Oleg, 1970 : « The Illustrated Maqâmât of the Thirteenth Century: the Bourgeoisie and the Arts ", in Albert H. Hourani \& Samuel M. STERN (éd.), The Islamic City, Oxford, p. 207-222.

GuidetTI, Mattia, 2009 : «The Byzantine Heritage in the Dār al-Islām: Churches and Mosques in al-Ruah between the sixth and Twelfth Centuries », Muqarnas 26, p. 1-36.

HAMPIIIAN, Nairy \& CYRAN, Monica, 1999: «Recent Discoveries in the Fatimid Palace Uncovered During the Conservation Works on Parts of al-Sâlihîyya Complex », in Marianne BARRUCAND (éd.), L'Égypte Fatimide, son art et son histoire, Actes du colloque des 28, 29 et 30 mai 1998, Paris, p. 669-683.

HeRzFeld, Ernst, 1953-1955 : Matériaux pour un Corpus Inscriptionum Arabicorum, Deuxième partie, Alep, 2 vol. (vol. 1 A, B, texte et vol. 2, planches), Le Caire (Mémoires de l'Institut français d'archéologie orientale 76-77).

HoffmAn, Eva R., 2000 : « The Beginnings of the Illustrated Arabic Book : an Intersection between Art and Scholarship », Muqarnas 17, p. 37-52.

Hunt, Lucy-Anne, 1991 : « Art and Colonialism, The Mosaics of the Church of the Nativity in Bethlehem (1169) and the Problem of "crusader" Art », Dumbarton Oaks Papers, p. 69-85.

Institut du Monde Arabe (IMA), 1998 : L'art des Fatimides, Catalogue d'exposition, Paris, 1998.

Institut du Monde Arabe (IMA), 2001 : L'Orient de Saladin, L'art des Ayyoubides, Catalogue d'exposition, Paris.

JAMES, David, 1974 : « Space-Forms in the Work of the Baghdâd "Maqâmât" Illustrators, 1225-58 ", Bulletin of the School of Oriental and African Studies 37, p. 305-320.

LAmPL, Paul, 1961 : «Schemes of Architectural Representation in Early Medieval Art », Marsyas 9, p. 6-13, et fig. 1-34.

PAUTY, Edmond, 1935: «L'architecture dans les miniatures islamiques », Bulletin de l'Institut d'Égypte, p. 25-68.

RabBAT, Nasser, 2003 : «The Dialogic Dimension of Umayyad Art », RES. Antropology and Aesthetics, 43, p. 78-94.

RabBat, Nasser, 1997-1999: "The Mosaics of the Qubba al-Zahiriyya in Damascus: A Classical Syrian Medium Acquires a Mamluk Signature », Aram 9-10, p. 1-13. 
RABY, Julian, 2004: «Nur al-Din, The Qastal a-Shu'aybiyya and the "Classical Revival” », Muqarnas 21 p. 289-310.

Rosen-Ayalon, Myriam, 2002 : Art et archéologie en Palestine, Paris, PUF.

SAUVAGET, Jean, 1932 : Monuments historiques de Damas, Beyrouth, Institut français de Damas.

SAUVAIRE, Henri, 1894-1896 : « Description de Damas », Journal Asiatique, IXe série, 3, 1894, p. 251-318 et 385$501 ; 4,1894$, p. 242-331 et 465-503; 5, 1895, p. 269-315 et 377-411; 6, 1895, p. 221-313 et 409-484; 7, 1896, p. $185-285$ et 369-459.

SERAJUdDin, Asma, 1975 : « The Representations of Architecture in the Manuscript Painting of the Abbassid Period », Journal of the Asiatic Society of Bangladesh 20/3, p. 31-40 et fig. 1-12.

SAUVAGET, Jean, 1938 : Les Monuments ayyoubides de Damas, I, Paris, De Boccard.

Sourdel, Dominique, Sourdel-Thomine, Janine, 1979 : « Dossier pour un corpus des inscriptions arabes de Damas », Revue des études islamiques 47-48, p. 119-173.

Soustiel, Jean : 1985 : La céramique islamique, Le guide du connaisseur, Paris, Office du Livre, p. 114-128.

TABBAA, Yasser, 1993 : « Survivals and Archaïsm in the Architecture of Northern Syria, ca. 1080- ca. 1150 », Muqarnas 10, p. 29-41.

TABBAA 2001 : The transformation of islamic Art during the Sunni Revival, Université de Washington.

VAN Berchem, Marguerite, 1969: «Mosaics of the Great Mosque of Umayyads in Damascus », in K. A. C. CRESWELL, Early Muslim Architecture, vol. I, part II, p. 323-372.

Velmans, Tania, 1964 : «Le rôle du décor architectural et la représentation de l'espace dans la peinture des Paléologues », Cahiers archéologiques 14, p. 183-216. 


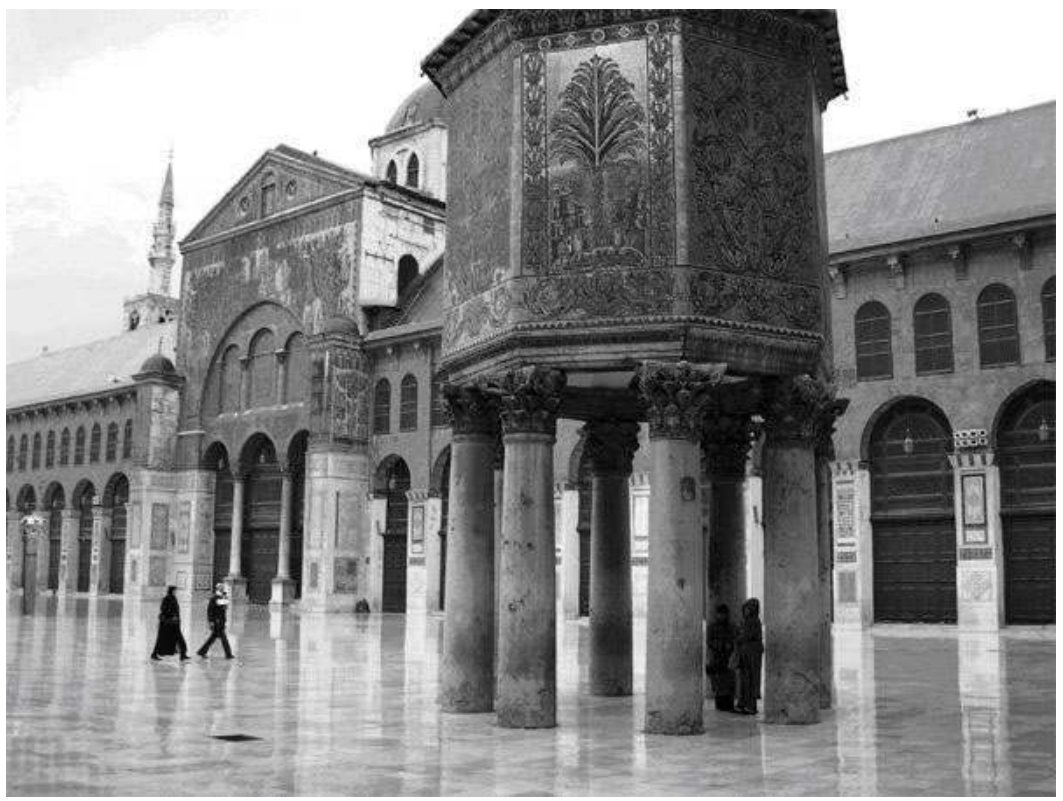

Fig. 1 - Vue d'ensemble de la façade extérieure de la salle de prière.

Fig. 2. Dessin repris et complété d'après M. ECOCHARD, 1937.

$<$ Ensemble A, «la mosquée $>\quad<$ Ensemble B $><\mathrm{C}><$ Ensemble D, « le palais »

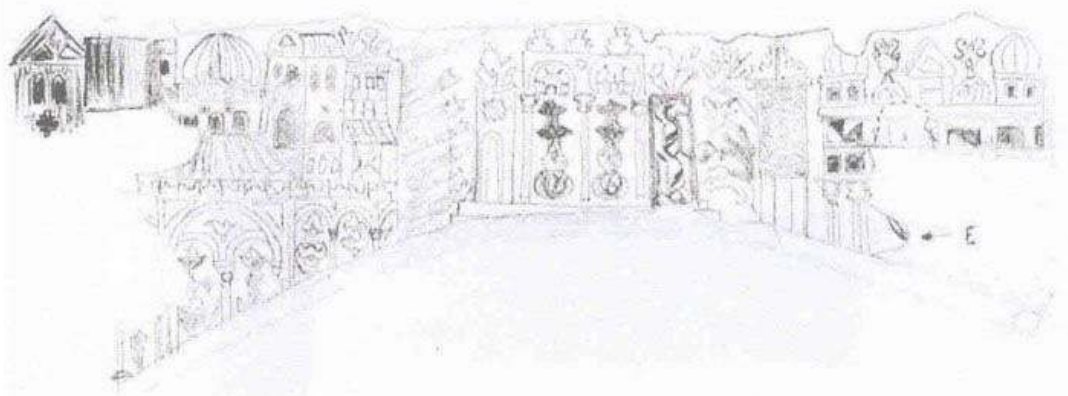

Détail 1 : merlons, toiture et grande arcature.

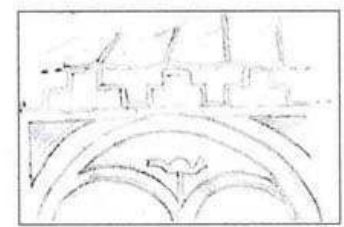

Détail 2 : « architrave » du palais.

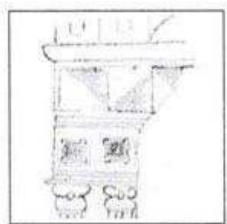

Fig. 2 - Dessin d'après Écochard, repris et complété. 


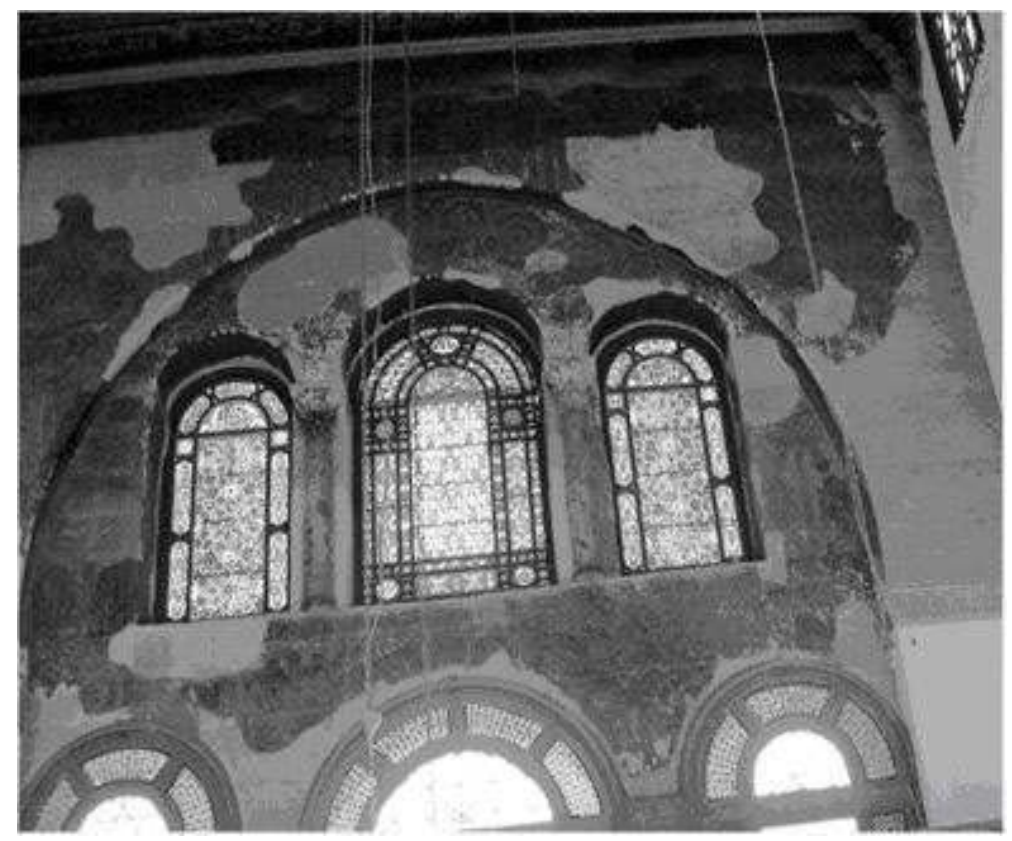

Fig. 3 - Vue d'ensemble du mur pignon à l'intérieur.

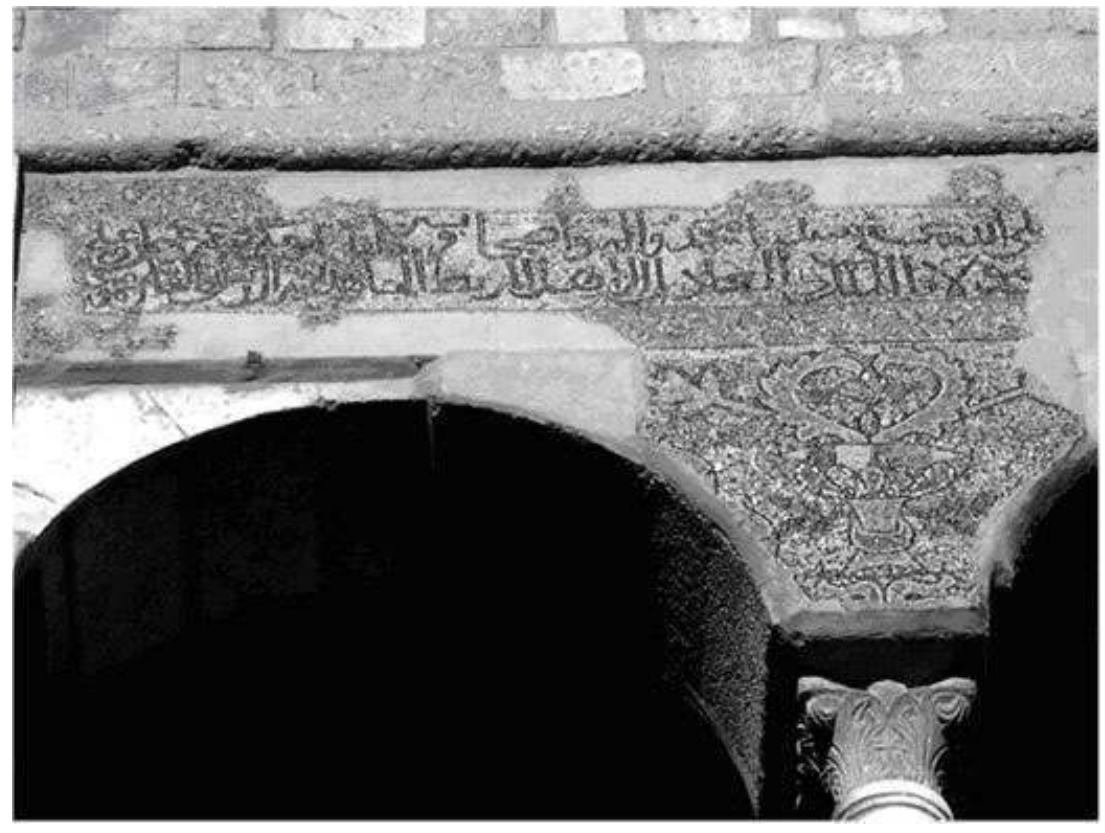

Fig. 4 - Inscription de Nūr al-Dīn près de l'angle nord-est de la cour. 


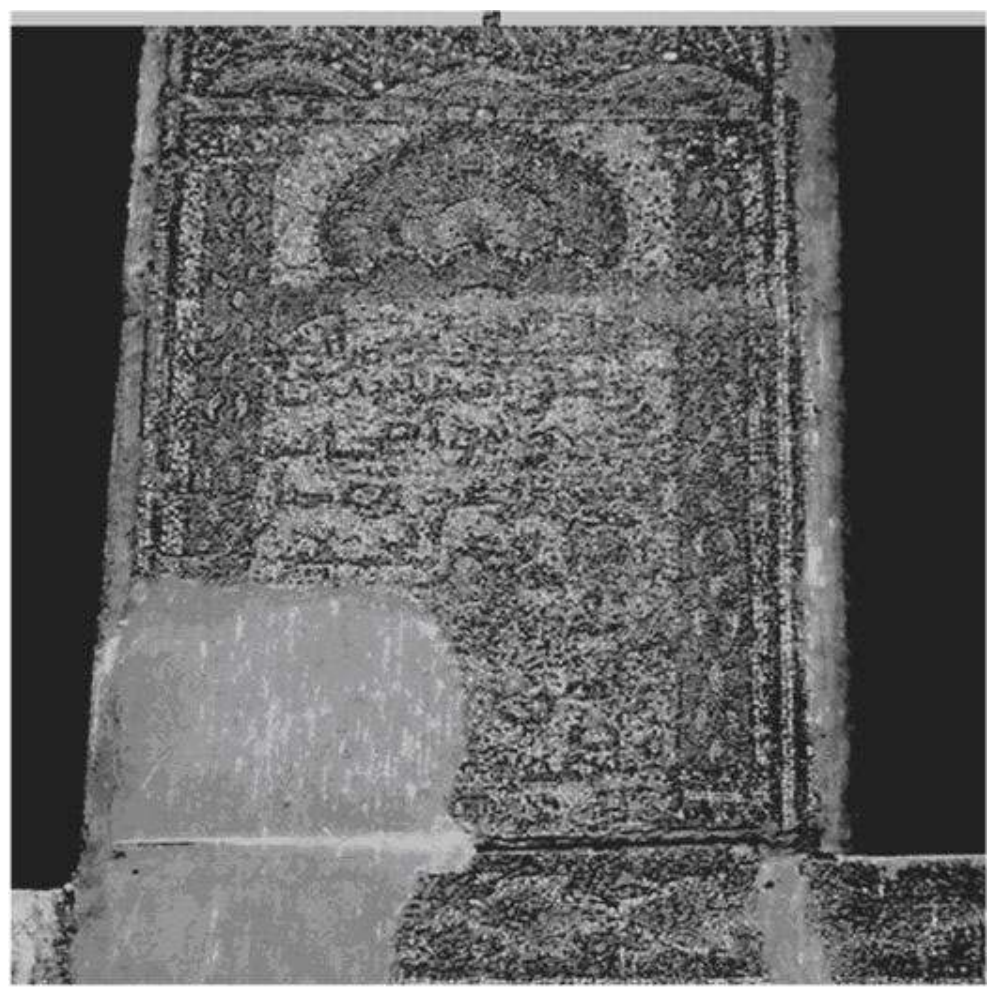

Fig. 5 - Portique nord, inscription du premier pilier à l'ouest de l'angle nord-est de la cour.

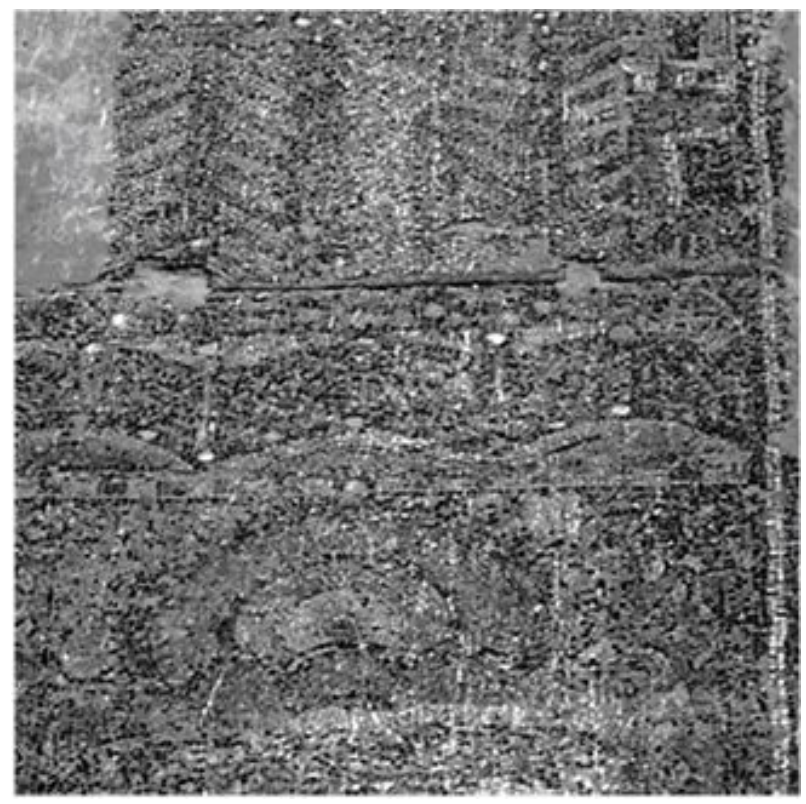

Fig. 6 - Détail du décor au-dessus de l’inscription précédente. 


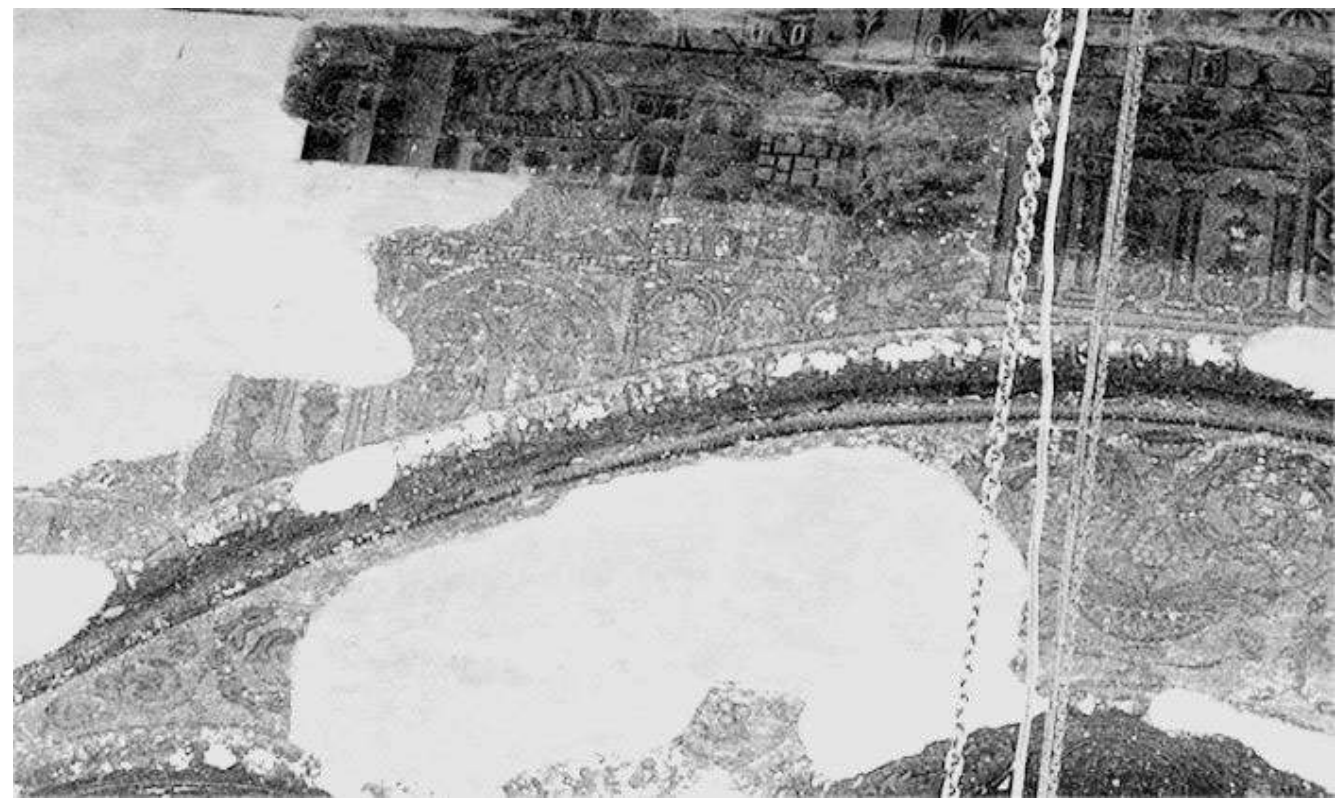

Fig. 7 - La mosquée, partie gauche de l'encadrement, zone A.

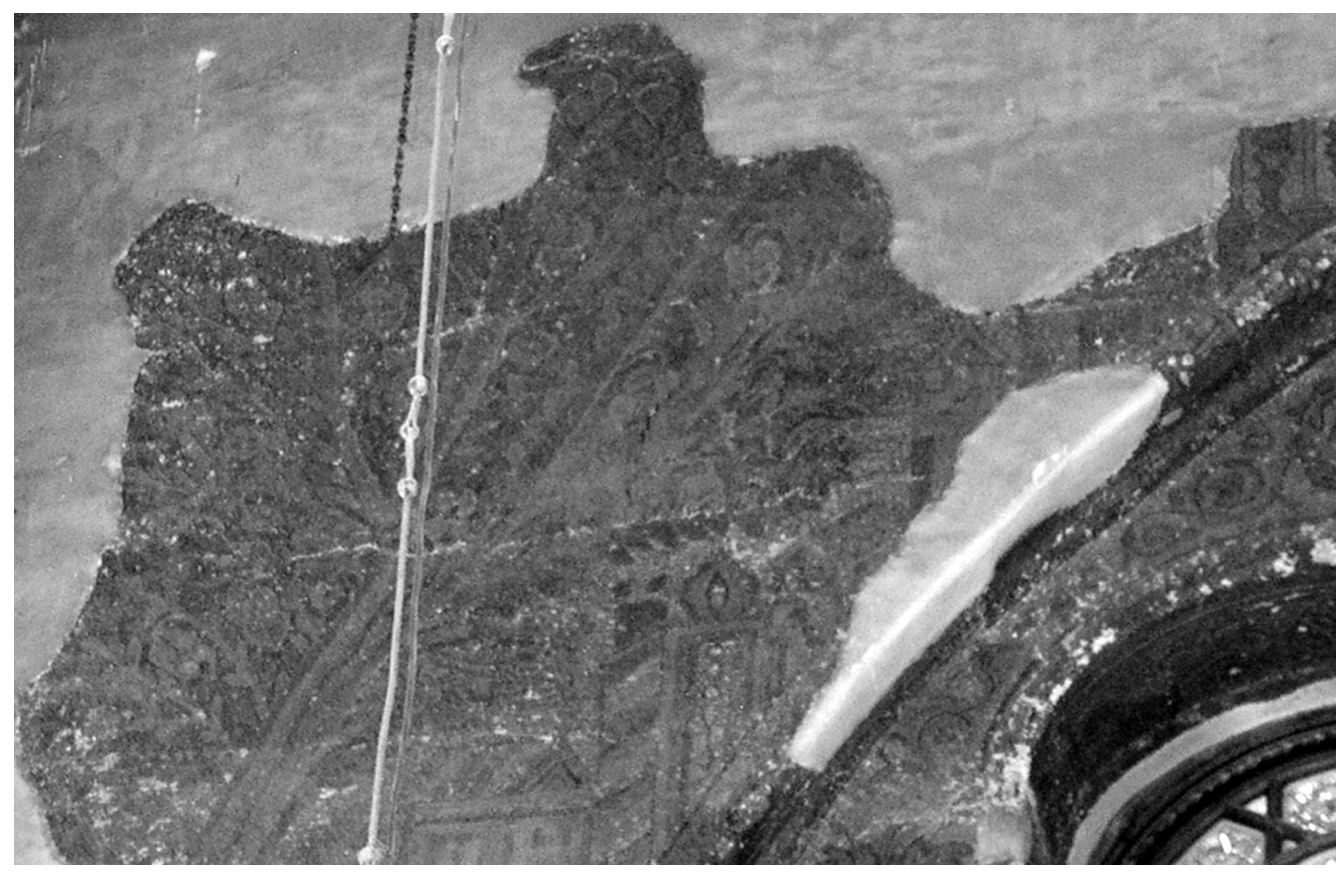

Fig. 8 - Ensemble F. Bas de l'encadrement, côté gauche. 


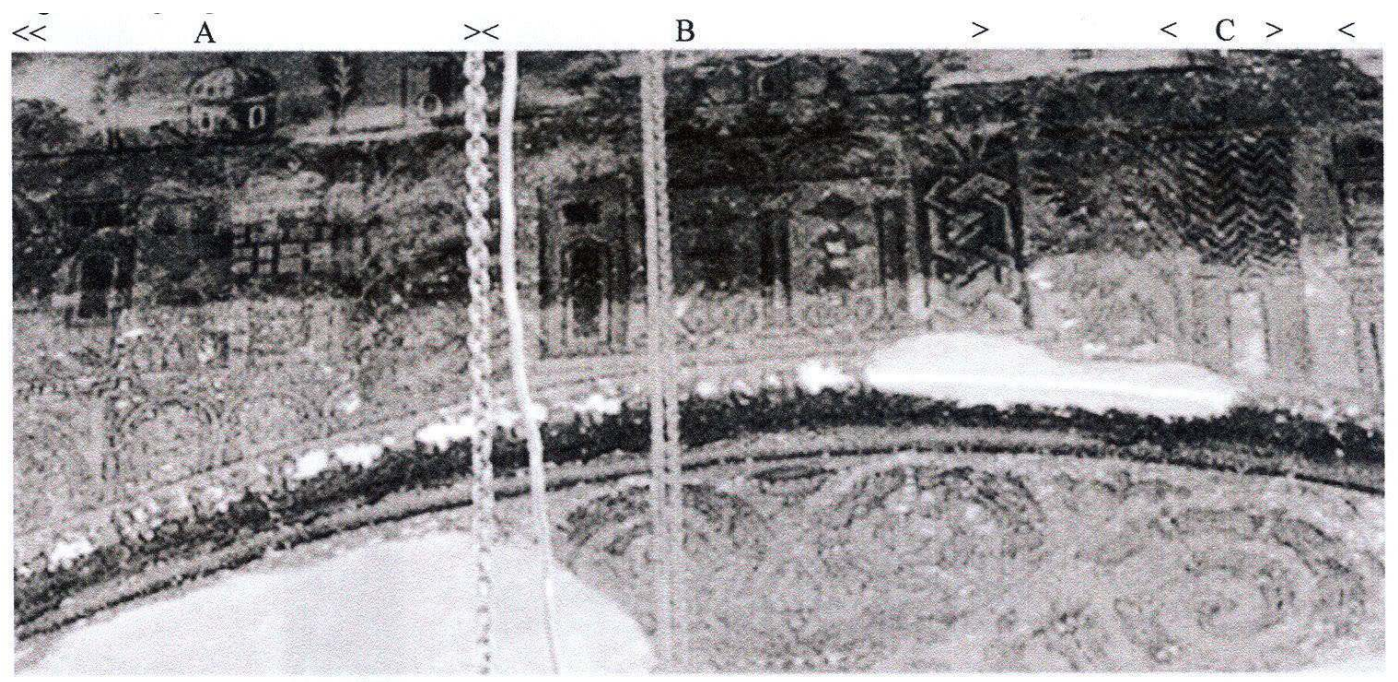

$\mathrm{D}$ :

Fig. 9 - Les groupes B et C.

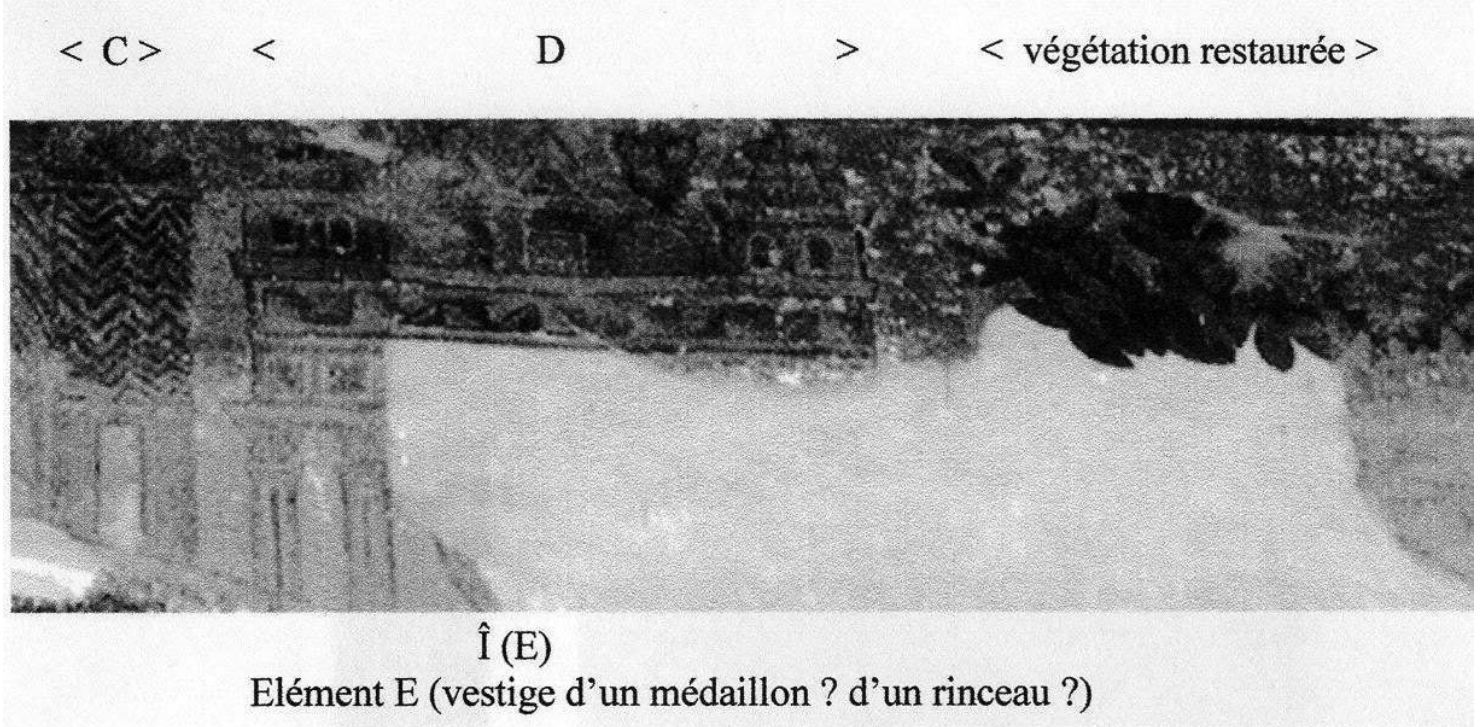

Fig. 10 - Le palais (D). 


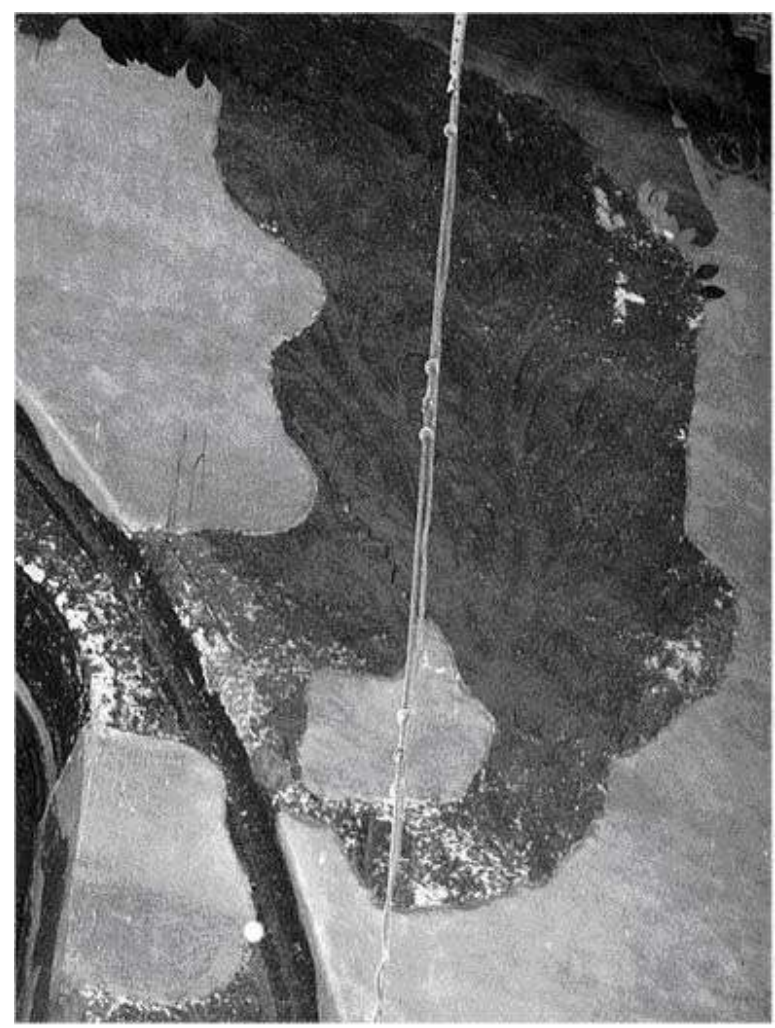

Fig. 11 - Partie droite de l'encadrement, feuillages.

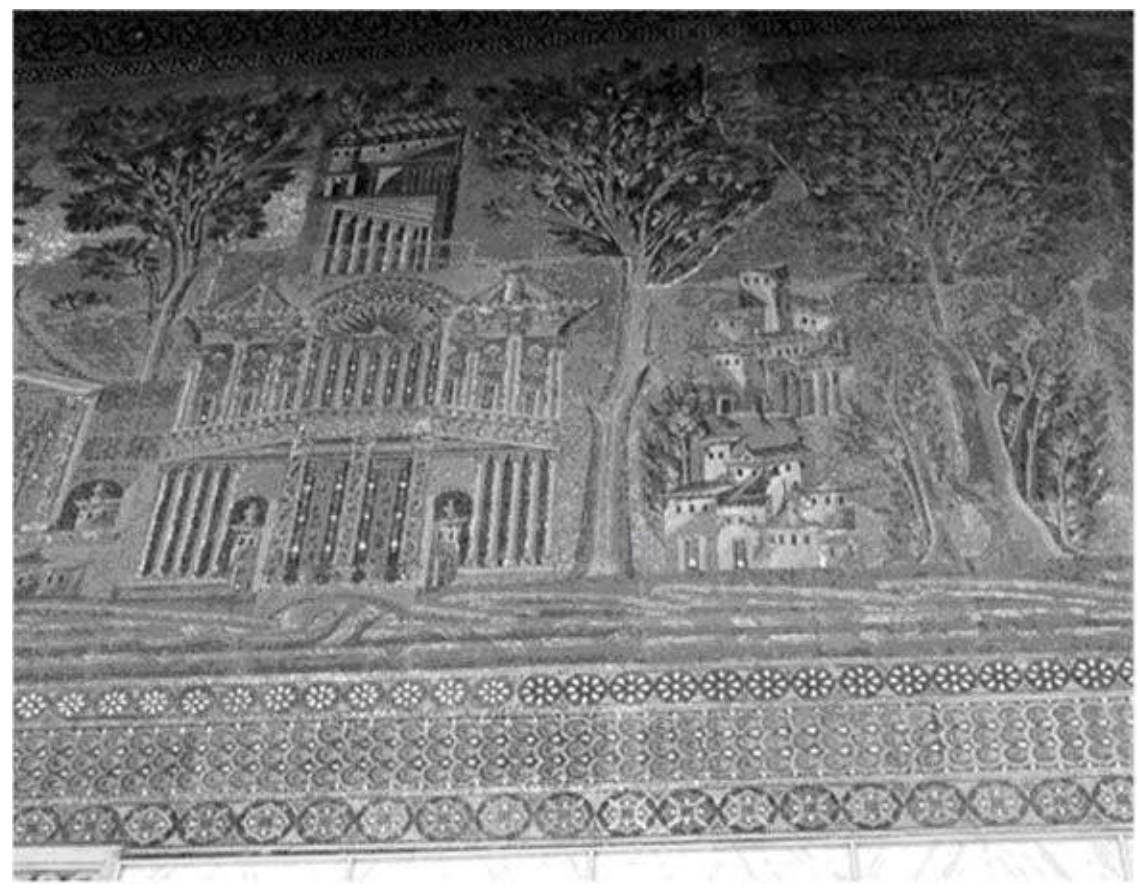

Fig. 12 - Panneau de mosaïque du portique occidental de la Grande Mosquée de Damas. Époque omeyyade. 


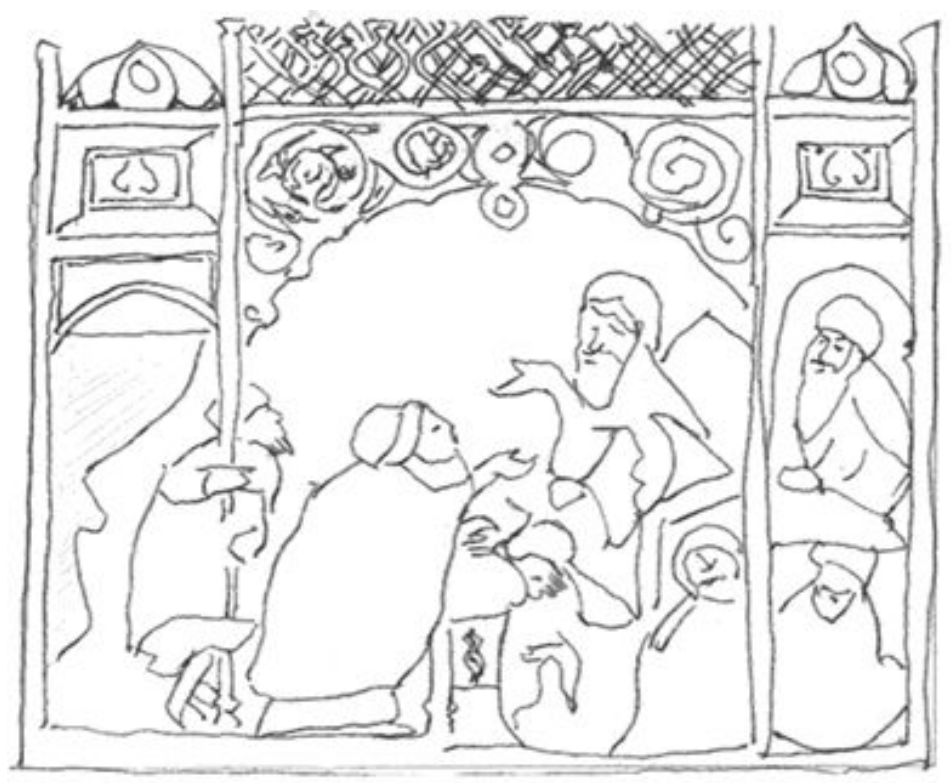

Fig. 13 - Maqāmāt d'al-Ḥarīrī, Qt-Petersbourg.

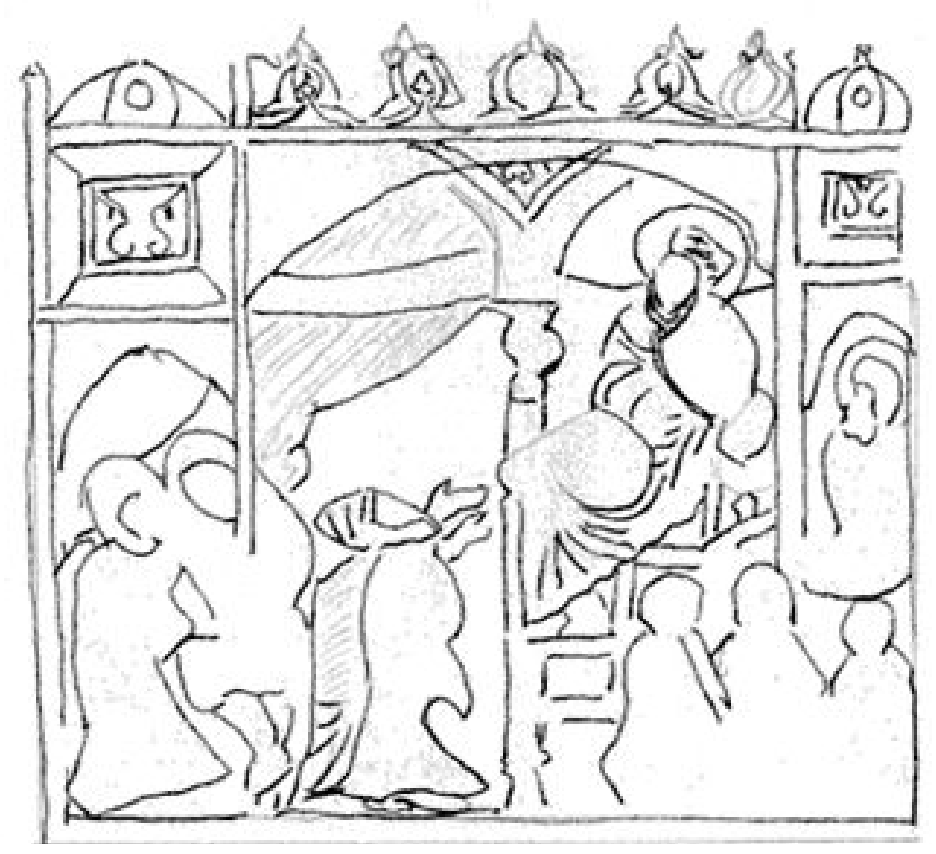

Fig. 14 - Maqāmāt d'al-Harīīī. 


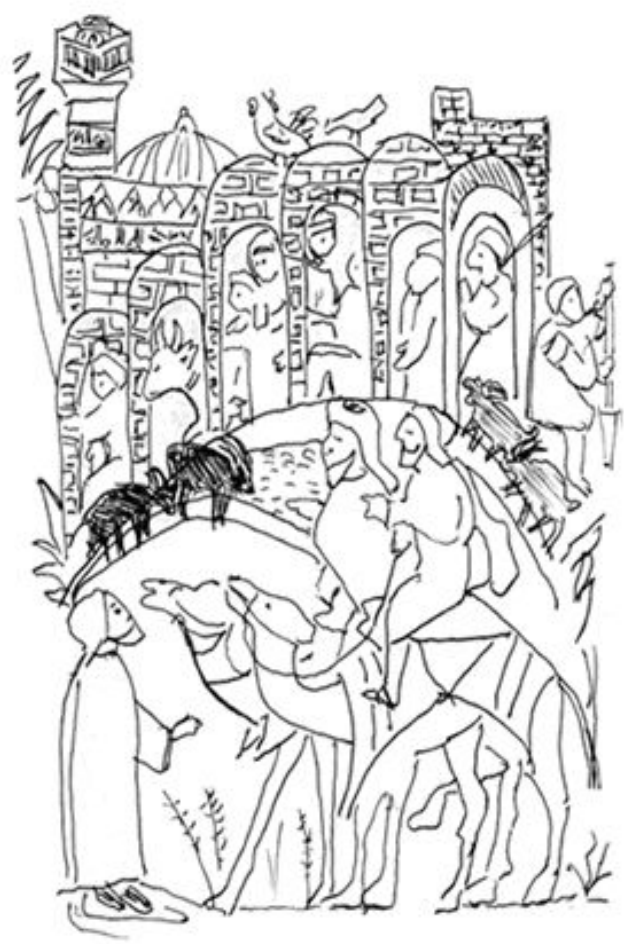

Fig. 15 - Maqāmāt d'al-Harīīī ; Ms. Schefer, fำ138.

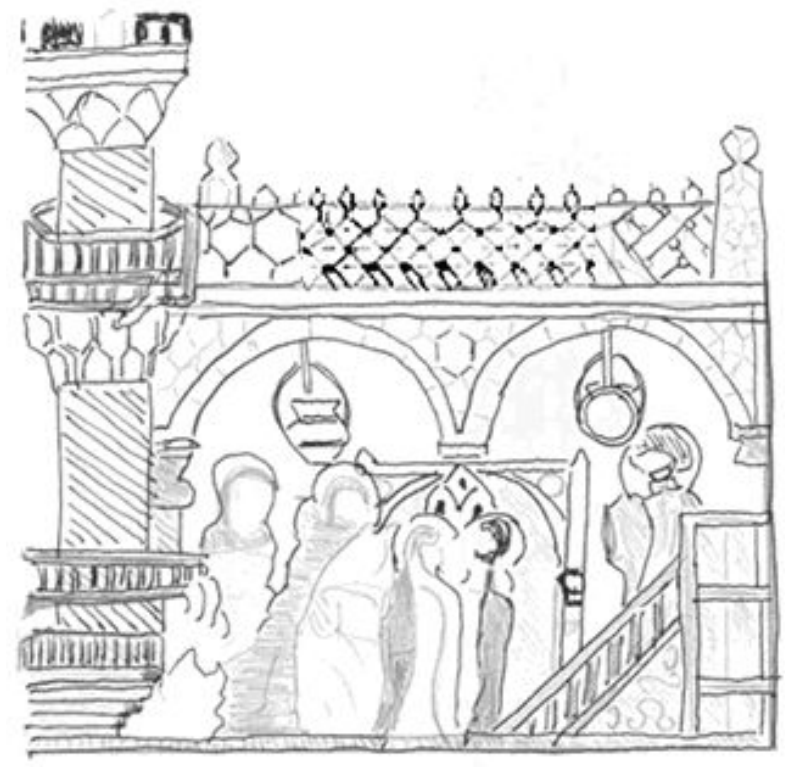

Fig. 16 - Maqāmāt d'al-Ḥarīrī, Istanbul. 


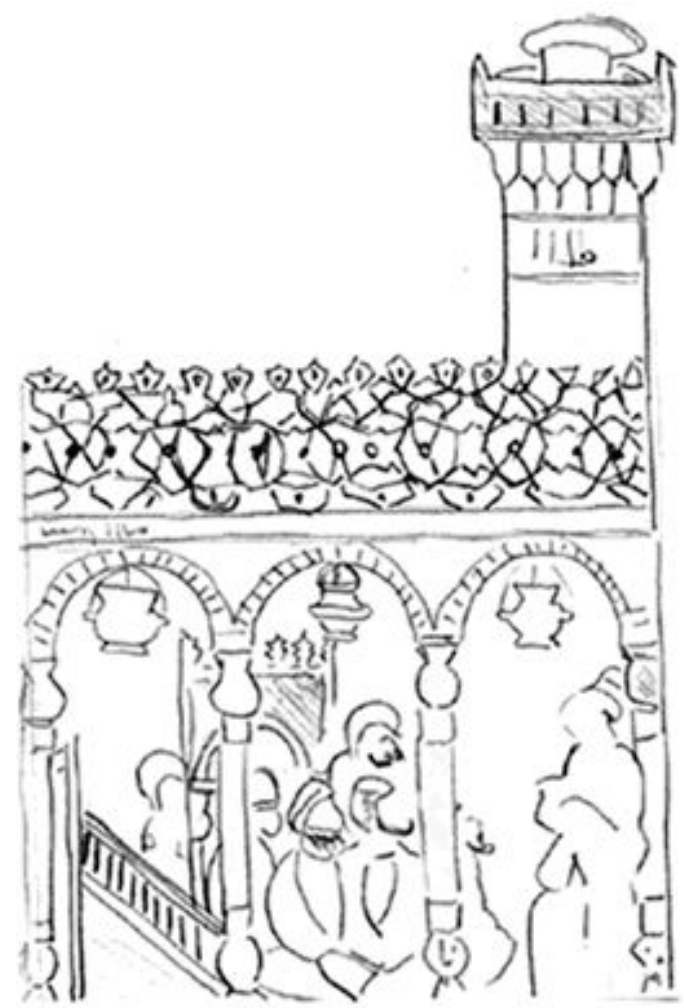

Fig. 17 - Maqāmāt d'al-Ḥarīrī, Schefer Paris.

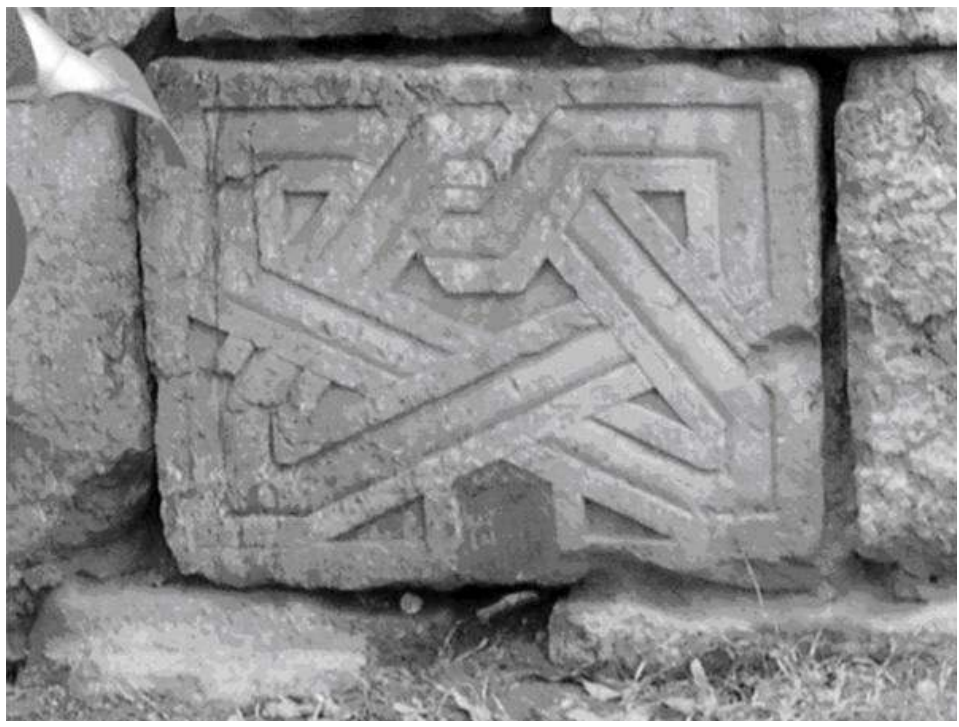

Fig. 18 - Décor rubané en noeud, pierre en remploi dans le mur nord de la citadelle de Damas. 


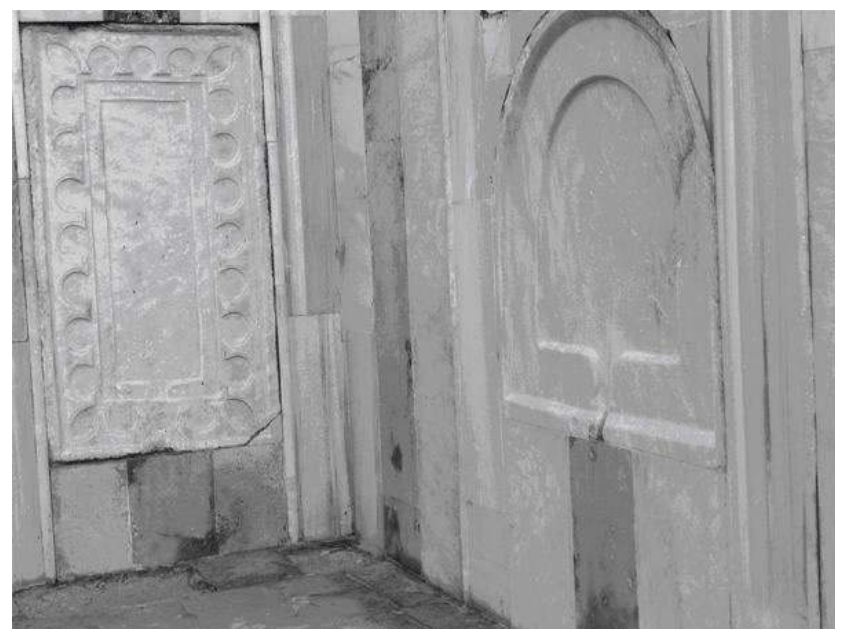

Fig. 19 - Tables de marbre en remploi dans le décor de l'īwān sud du Māristān de Nūr al-Dīn.

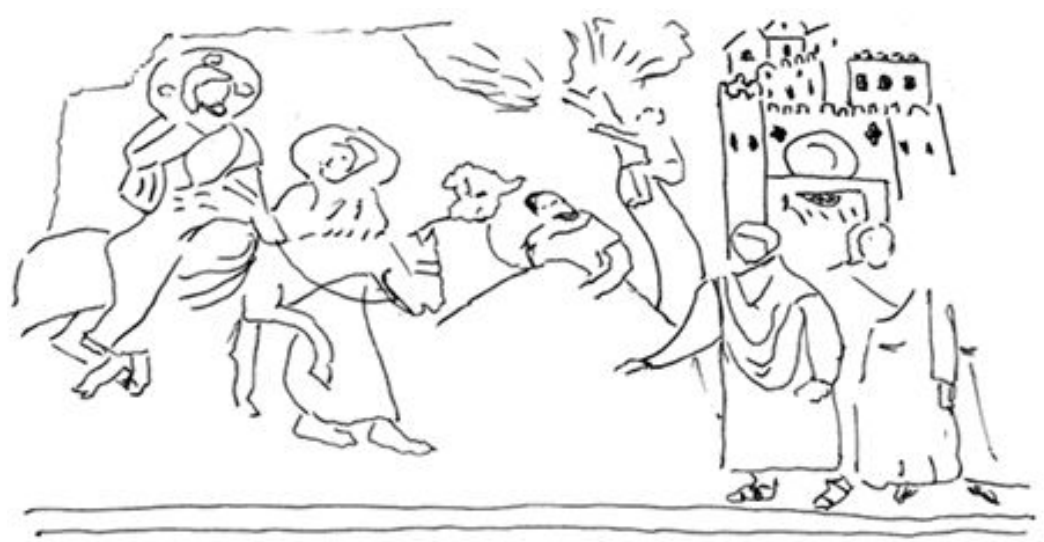

Fig. 20 - Bethléem, décor de mosaïque, Église de la Nativité, 1165-1169. Croquis Bogard d'après le cliché Franciscan Cyberspot.

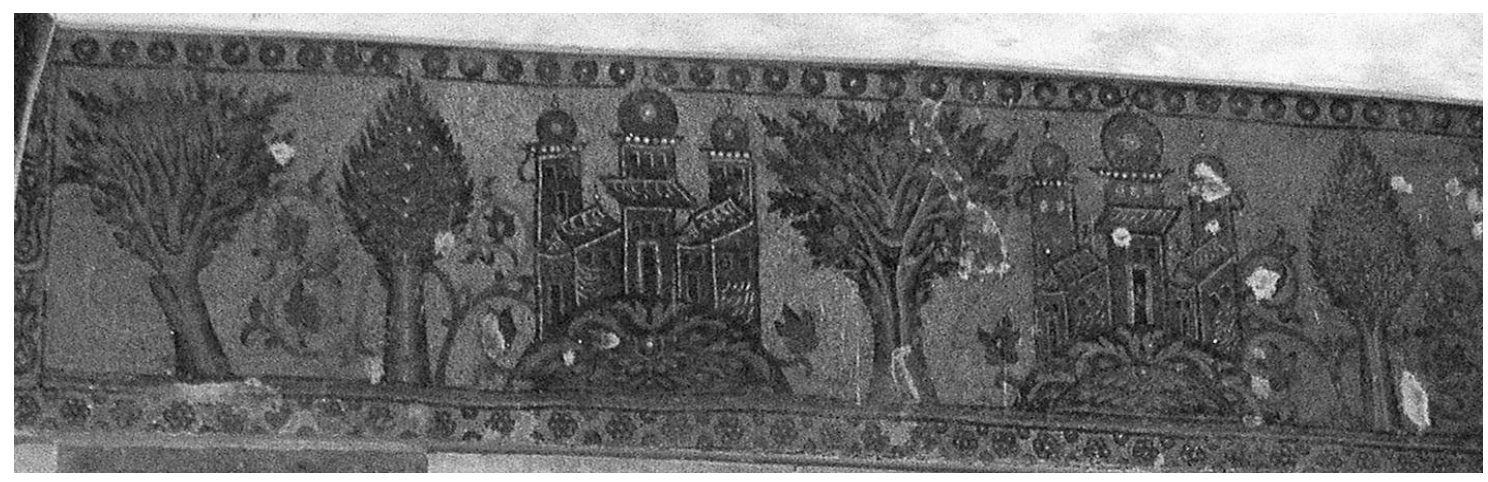

Fig. 21 - Panneau de mosaïque du Mausolée de Baybars. Paroi occidentale. Bogard, 2007. 